\title{
Semi-global leader-following output consensus of heterogeneous systems subject to actuator position and rate saturation
}

\author{
Panpan Zhou* ${ }^{*}$ and Ben M. Chen
}

\begin{abstract}
We study in this paper a semi-global leader-following output consensus problem for multiple heterogeneous linear systems in the presence of actuator position and rate saturation over a directed topology. For each follower, via the low gain feedback design technique and output regulation theory, both a state feedback consensus protocol and an output feedback consensus protocol are constructed. In the output feedback case, different distributed observers are designed for the informed followers and uninformed followers to estimate the state of the leader and the follower itself. We show that the semi-global leader-following output consensus of heterogeneous linear systems can be achieved by the two consensus protocols if each follower is reachable from the leader in the directed communication topology.
\end{abstract}

Keywords: Multi-agent system, Output consensus, Low gain feedback

\section{Introduction}

Consensus control, a fundamental problem of cooperative control for multi-agent systems, entails the construction of control protocols for every agent so that the states/outputs of all agents converge to an agreement value when there is no leader agent (see Radenković and Krstić [1], Wang et al. [2], Meng et al. [3] and Li et al. [4]), and the states/outputs of all followers converge to the state/output of the leader if there is one leader (see Dong et al. [5] and Lu and Liu [6]). In real applications with substantial number of agents, to reduce communication pressure, distributed control protocols are preferred, that is, only local information is used.

In the early literature, many results are obtained in the consensus problem of a multi-agent system. With static communication graph, $\mathrm{Li}$ et al. solved the problem for general linear systems with adaptive dynamic protocols [7] and fully distributed control laws [8]. Ma and Miao

${ }^{*}$ Correspondence: ppzhou@link.cuhk.edu.hk

Department of Mechanical and Automation Engineering, The Chinese

University of Hong Kong, Shatin, N.T., Hong Kong
[9] and Han et al. [10] focused on the output consensus of heterogeneous linear systems via an output regulation approach. The consensus problem of a class of linear firstorder, second-order together with Euler-Lagrange systems were considered by Liu et al. [11], a class of nonlinear systems by Liu and Huang [12] and rigid bodies by Yu et al. [13]. It is well noted that actuator saturation is ubiquitous in practical control systems, however, these early results do not take it into consideration.

In recent years, there are some results on the semiglobal consensus problem of a group of multi-agent system with actuator saturation (see [14-19]). In view of input saturation, low gain feedback design technique (see Lin [20]) is of great significance in guaranteeing the control input to remain unsaturated by tuning the low gain parameter small enough, given any arbitrarily large and bounded set of initial conditions. Via the low gain feedback design technique, the semi-global output containment control or bipartite consensus of multiple linear systems (see $[15,16]$ ) and multiple heterogeneous linear systems (see Shi et al. [17]) are solved, if the interaction topology is structurally balanced and contains a 
spanning tree. The discrete-time counterparts of these results are also obtained in $[18,19]$. There are also some results on global consensus problem of multi-agent systems subject to actuator saturation. In particular, Zheng et al. [21] constructed a saturated consensus protocol for each second-order follower system under undirected connected graphs, but the input bound depends on the communication graph, which may not meet the requirements of real systems. It is shown by Meng et al. [22] that for neurally stable systems and double integrator systems, the global leader-following consensus can be achieved by linear local feedback laws over a static communication topology and nonlinear local feedback laws over a switching topology. As an extension of the results done by Meng et al. [22], Xie and Lin [23] constructed a bounded consensus protocol with intermittent directed communication to solve the global leader-following consensus problem for a group of agents described by a chain of integrators of an arbitrary length.

Besides the position saturation of actuators, actuator rate saturation may worsen the performance of the closedloop system, and may even lead to instability. As reported in [24], actuator saturation is exactly a contributing factor for the mishaps of YF-22 fighter aircraft. Therefore, it is crucial to take into account both the actuator position and rate saturation in the consensus problem for a multi-agent system. The position and rate-limited case is firstly studied by Lin [25] to solve the semi-global stabilization problem of a linear system if the open-loop system is stabilizable and all its poles located at the closed lefthalf complex plane. Lim and Ahn [26] are for a class of nonlinear interconnected systems where a decentralized state feedback controller is proposed based on linear matrix inequality conditions. In recent years, the methods of Lin [25] are extended to the coordination control of multiple linear systems subject to actuator position and rate saturation. The semi-global containment control and leader-following consensus problems are, respectively, considered by Zhao and Lin [27], and Zhao and Shi [28], where both state feedback control and output feedback control are proposed under connected undirected graphs.

To the best of our knowledge, there is no result on the output consensus problem for multiple heterogeneous systems with both actuator position and rate saturation, which is exactly the problem we consider in this paper. By the low gain approach and output regulation theory, we construct both a state feedback consensus protocol and output feedback consensus protocol for each follower over a directed network. In the state feedback type, the protocol is designed based on a distributed observer that estimates the state of the leader. In the output feedback type, different distributed observers that estimate the state of the leader and the follower itself are designed for the informed followers and uninformed followers which respectively have and do not have access to the output of the leader. It is worthy to note that the methods in the consensus or containment control for linear systems in $[27,28]$ can not be used to solve the consensus of heterogeneous systems by a simple modification under output regulation theory. The nontrivial consensus protocols we designed allow the actuator rate converge to the value it should be when the consensus problem is solved. Moreover, our consensus protocols are applicable to directed topologies.

The outline of the rest of this article is as follows. Section 2 gives the definitions of both semi-global state feedback type and output feedback type leader-following output consensus of heterogeneous linear systems with position and rate-limited actuators. Two corresponding consensus protocols are respectively constructed in Subsections 3.1 and 3.2. We give illustrative examples in Section 4 to verify the effectiveness of the two control laws. Finally, we conclude our work by Section 5 with some remarks.

Throughout this paper, for a time constant $T \geq 0$ and a signal $x: \mathbb{R}_{+} \rightarrow \mathbb{R}^{s}, x=\left[x_{1}, x_{2}, \cdots, x_{s}\right]^{\mathrm{T}},|x|$ denotes the Euclidean norm, $\|x\|_{\infty}=\max _{i}\left|x_{i}\right|$, and $\|x\|_{T, \infty}=$ $\sup _{t \geq T}|x| . \mathbf{1}_{N} \in \mathbb{R}^{N}$ denotes a vector with all elements being $1 . I_{n} \in \mathbb{R}^{n \times n}$ is the identity matrix. Kronecker product is denoted by $\otimes . X^{\mathrm{T}}$ stands for the transpose of the vector or matrix $X .0$ represents a vector or matrix of zero with appropriate dimension.

\section{Problem formulation and preliminaries}

Consider a group of $N+1$ heterogeneous systems consisting of a leader and $N$ followers. The leader, labeled as 0 , is described as

$$
\left\{\begin{array}{c}
\dot{w}=S w, \\
y_{0}=-Q w,
\end{array}\right.
$$

where $w \in \mathbb{R}^{s}, y_{0} \in \mathbb{R}^{m}$ are the state and output, respectively. Similar to the system in [29], the dynamics of the $i$-th follower, $i=1,2 \cdots, N$, is subject to actuator position and rate saturation and it is described by the following equation,

$$
\left\{\begin{array}{l}
\dot{x}_{i}=A_{i} x_{i}+B_{i} \sigma_{p}\left(v_{i}\right)+W_{i} w, \\
\dot{v}_{i}=\sigma_{r}\left(-\bar{T}_{i} v_{i}+\bar{T}_{i} u_{i}\right), \\
y_{i}=C_{i} x_{i}, \\
e_{i}=C_{i} x_{i}+Q w, i=1,2, \cdots, N,
\end{array}\right.
$$

where $x_{i} \in \mathbb{R}^{n_{i}}, y_{i} \in \mathbb{R}^{m}$ and $u_{i} \in \mathbb{R}^{q_{i}}$ are respectively, the plant state, output and control input of the $i$-th follower. The second equation denotes the actuator dynamics with state $v_{i} \in \mathbb{R}^{q_{i}}$. The positive definite diagonal matrices $\bar{T}_{i}=\operatorname{diag}\left\{\tau_{i, 1}, \tau_{i, 2}, \cdots, \tau_{i, q_{i}}\right\} \in \mathbb{R}^{q_{i} \times q_{i}}$ represents the "time constants" of the actuators. $e_{i} \in$ 
$\mathbb{R}^{m}$, which is called the regulated output, denotes output tracking error between the $i$-th follower and the leader. $W_{i} w$ with $W_{i} \in \mathbb{R}^{n_{i} \times n}$ represents external disturbances caused by the leader. $\sigma_{p}(\cdot), \sigma_{r}(\cdot): \mathbb{R}^{q_{i}} \rightarrow \mathbb{R}^{q_{i}}$ represent vector valued saturation functions with $p$ and $r$ are known constants. For $v_{i}=\left[v_{i 1}, v_{i 2}, \cdots, v_{i q_{i}}\right]^{\mathrm{T}}, \sigma_{p}\left(v_{i}\right)=$ $\left[\sigma_{p}\left(v_{i 1}\right), \sigma_{p}\left(v_{i 2}\right), \cdots, \sigma_{p}\left(v_{i q_{i}}\right)\right]^{\mathrm{T}}$. For each $j=1,2, \cdots, q_{i}$, $\sigma_{p}\left(v_{i j}\right)=\operatorname{sgn}\left(v_{i j}\right) \min \left\{\left|v_{i j}\right|, p\right\}$ is the standard saturation function.

In this paper, we aim to design a distributed control law $u_{i}$ for each follower so that the output tracking error satisfies $\lim _{t \rightarrow \infty} e_{i}=0$.

The communication topology among the leader and the followers is represented by a directed graph $\mathcal{G}=\{\mathcal{V}, \mathcal{E}\}$, with $\mathcal{V}=\{0,1, \cdots, N\}$ being the node set and $\mathcal{E}=\mathcal{V} \times \mathcal{V}$ being the edge set. For $i, j \in \mathcal{V},(j, i) \in \mathcal{E}$ if and only if node $i$ have access to the information of node $j$. Then node $j$ is called the neighbor of node $i$ and node $i$ is called a child of node $j$. We use $\mathcal{F}=\{1,2, \cdots, N\}$ to denote the set of followers, and use $\mathcal{N}_{i}:=\{j:(j, i) \in \mathcal{E}\}$ to represent the set of neighbors of node $i$. Depending on whether or not the followers have access to the information of the leader, the followers are divided into two classes. The informed ones can obtain the information of the leader, while the uninformed ones can not, and we use $\mathcal{F}_{\text {in }}$ and $\mathcal{F}_{\text {un }}$ to represent the informed followers and the uninformed followers, respectively, that is, $\mathcal{F}_{i n}:=\{i: i \in \mathcal{F},(0, i) \in \mathcal{E}\}$ and $\mathcal{F}_{\text {un }}:=\mathcal{F} \backslash \mathcal{F}_{\text {in }}$. Without loss of generality, we assume the first $l$ followers are the informed ones and the left $N-l$ followers are the uninformed ones. If the graph contains a sequence of edges $\left(i_{1}, i_{2}\right),\left(i_{2}, i_{3}\right), \cdots,\left(i_{k-1}, i_{k}\right)$, then we say there is a directed path from node $i_{1}$ to $i_{k}$, or $i_{k}$ is reachable from $i_{1}$. For a directed graph $\mathcal{G}$, the adjacency matrix $\mathcal{A}=\left[a_{i j}\right] \in \mathbb{R}^{(N+1) \times(N+1)}$ is defined as $a_{i j}=1$ if $(j, i) \in \mathcal{E}$, otherwise, $a_{i j}=0$. The Laplacian matrix $\mathcal{L}=$ $\left[l_{i j}\right] \in \mathbb{R}^{(N+1) \times(N+1)}$ is defined as $l_{i j}=-a_{i j}$ if $i \neq j$, and $l_{i i}=\sum_{j=0}^{N} a_{i j}$. According to the classification of the leader, the informed followers and the uninformed followers, $\mathcal{L}$ can be partitioned as

$$
\mathcal{L}=\left[\begin{array}{ll}
0 & \mathbf{0} \\
\mathcal{L}_{f l} & \mathcal{L}_{f f}
\end{array}\right]=\left[\begin{array}{lll}
0 & \mathbf{0} & \mathbf{0} \\
\mathcal{L}_{1} & \mathcal{L}_{2} & \mathcal{L}_{3} \\
\mathbf{0} & \mathcal{L}_{4} & \mathcal{L}_{5}
\end{array}\right]
$$

where $\mathcal{L}_{f l} \in \mathbb{R}^{N \times 1}, \mathcal{L}_{f f} \in \mathbb{R}^{N \times N}, \mathcal{L}_{1} \in \mathbb{R}^{l \times 1}, \mathcal{L}_{2} \in \mathbb{R}^{l \times l}$, $\mathcal{L}_{3} \in \mathbb{R}^{l \times(N-l)}, \mathcal{L}_{4} \in \mathbb{R}^{(N-l) \times l}$ and $\mathcal{L}_{5} \in \mathbb{R}^{(N-l) \times(N-l)}$.

Assumption 1 There is a directed path from the leader node 0 to each follower node $i$.

Assumption 2 The following regulator equations

$$
\begin{aligned}
& \Pi_{i} S=A_{i} \Pi_{i}+B_{i} \Gamma_{i}+W_{i}, \\
& C_{i} \Pi_{i}+Q=0, \quad i=1,2, \ldots, N
\end{aligned}
$$

have a pair of solutions $\Pi_{i} \in \mathbb{R}^{n_{i} \times s}$ and $\Gamma_{i} \in \mathbb{R}^{q_{i} \times s}$.

Assumption 3 For each $i=1,2, \cdots, N$, the pair $\left(A_{i}, B_{i}\right)$ is stabilizable, and all eigenvalues of $A_{i}$ have non-positive real parts.

Assumption 4 For each $i=1,2, \cdots, N$, the pair $(Q, S)$ is detectable.

Lemma 1 [Lin [20]] Suppose Assumption 3 holds. For each $\epsilon \in(0,1]$, there exists a unique positive definite matrix $P_{i}(\epsilon) \in \mathbb{R}^{n_{i} \times n_{i}}$ of the following parametric algebraic Riccati equation (ARE):

$$
A_{i}^{\mathrm{T}} P_{i}(\epsilon)+P_{i}(\epsilon) A_{i}-\gamma P_{i}(\epsilon) B_{i} B_{i}^{\mathrm{T}} P_{i}(\epsilon)=-\epsilon I_{n_{i}},
$$

where $\gamma$ is a positive constant. In addition, $\lim _{\epsilon \rightarrow 0} P_{i}(\epsilon)=$ 0 .

Assumption 5 For each $i=1,2, \cdots, N$, there exist a time $T \geq 0$ and two positive constants $\delta_{p}$ and $\delta_{r}$, such that $0<p-\left\|\Gamma_{i} w\right\|_{T, \infty} \leq \delta_{p}$ and $0<r-\left\|\Gamma_{i} S w\right\|_{T, \infty} \leq \delta_{r}$ for all $w$ with $w(0) \in \mathcal{W}_{0}$, where $\mathcal{W}_{0}$ is a priori given bounded set.

Remark 1 Assumption 5 means that $w$ is bounded for all time $t \geq T$. Since $w$ is determined by (1), it implies that all eigenvalues of $S$ have non-positive real parts, and those eigenvalues with zero real parts are semi-simple. Besides, Assumption 5 also implies $p>\left\|\Gamma_{i} w\right\|_{T, \infty}$ and $r>$ $\left\|\Gamma_{i} S w\right\|_{T, \infty} . \Gamma_{i} w$ and $\Gamma_{i} S w$ can be viewed as the generalized actuator position and rate of the leader. If the actuator position or rate of each follower is less than that of the leader, i.e., $p<\left\|\Gamma_{i} w\right\|_{T, \infty}$ or $r<\left\|\Gamma_{i} S w\right\|_{T, \infty}$, it is impossible for the followers to catch up the leader when it moves at its maximal pace. Thus, Assumption 5 is reasonable in real applications.

For the case that the states of all agents can be measured, a distributed observer is firstly designed for each follower to estimate the state of the leader. Consider the following distributed observer:

$$
\dot{\eta}_{i}=S \eta_{i}+\mu_{1}\left(\sum_{j=1}^{N} a_{i j}\left(\eta_{j}-\eta_{i}\right)+a_{i 0}\left(w-\eta_{i}\right)\right), \quad i \in \mathcal{F},
$$

where $\mu_{1}$ is a positive constant such that $\left(I_{N} \otimes S-\mu_{1} \mathcal{L}_{f f} \otimes\right.$ $\left.I_{s}\right)$ is Hurwitz. Such a $\mu_{1}$ exists because under Assumption 1 , all eigenvalues of $\mathcal{L}_{f f}$ have positive real parts (see the work of Hong et al. [30]).

The state feedback-based semi-global leader-following output consensus problem formed by followers (2) and leader (1) is defined as follows. 
Problem 1 (State feedback-based semi-global leaderfollowing output consensus problem)

Consider a multi-agent system consisting of leader (1) and followers (2). Assume that Assumptions 1-3 and 5 hold. Let $x=\left[x_{1}^{\mathrm{T}}, \cdots, x_{N}^{\mathrm{T}}\right]^{\mathrm{T}}, v=\left[v_{1}^{\mathrm{T}}, \cdots, v_{N}^{\mathrm{T}}\right]^{\mathrm{T}}, \eta=\left[\eta_{1}^{\mathrm{T}}, \cdots, \eta_{N}^{\mathrm{T}}\right]^{\mathrm{T}}$, $n=n_{1}+n_{2}+\cdots+n_{N}$, and $q=q_{1}+q_{2}+\cdots+q_{N}$. For a priori given bounded sets $\mathcal{X}_{0} \subset \mathbb{R}^{n}, \mathcal{V}_{0} \subset \mathbb{R}^{q}$, $\mathcal{W}_{0} \subset \mathbb{R}^{s}$ and $\mathcal{Z}_{0} \subset \mathbb{R}^{N s}$, construct a state feedback consensus protocol $u_{i}=f_{i}\left(x_{i}, v_{i}, \eta_{i}\right)$ for each follower, based on the distributed leader state observer (5), such that for $\left[x^{\mathrm{T}}(0), v^{\mathrm{T}}(0), w^{\mathrm{T}}(0), \eta^{\mathrm{T}}(0)\right]^{\mathrm{T}} \in \mathcal{X}_{0} \times \mathcal{V}_{0} \times \mathcal{W}_{0} \times \mathcal{Z}_{0}$, the leader-following output consensus is achieved, that is, for any $i \in \mathcal{F}$,

$$
\lim _{t \rightarrow \infty} e_{i}=0 .
$$

However, in reality, the plant state information of the agents may not be available, while only the outputs can be measured. In such a case, output-based estimations of the states of the leader and followers should be designed for each follower. To this end, consider the following dynamic compensators:

$$
\begin{aligned}
& \dot{\hat{\hat{w}}}_{i}=S \hat{w}_{i}+L_{S, i}\left(y_{0}+Q \hat{w}_{i}\right), i=1,2, \cdots, l, \\
& \dot{\hat{w}}_{i}=S \hat{w}_{i}+\mu_{2} \sum_{j=1}^{N} a_{i j}\left(\hat{w}_{j}-\hat{w}_{i}\right), i=l+1, \cdots, N, \\
& \dot{\hat{x}}_{i}=A_{i} \hat{x}_{i}-L_{A, i}\left(y_{i}-C_{i} \hat{x}_{i}\right)+W_{i} \hat{w}_{i}, i=1,2, \cdots, N,
\end{aligned}
$$

where $\hat{x}_{i} \in \mathbb{R}^{n_{i}}$ and $\hat{w}_{i} \in \mathbb{R}^{s}$ are respectively designed to estimate the state of the $i$-th follower itself and the state of the leader. $L_{S, i}$ and $L_{A, i}$ are observer gains such that $S+$ $L_{S, i} Q$ and $A_{i}+L_{A, i} C_{i}$ are Hurwitz. $\mu_{2}$ is a positive constant such that $\left(I_{N-l} \otimes S-\mu_{2} \mathcal{L}_{5} \otimes I_{S}\right)$ is Hurwitz. Such a $\mu_{2}$ exists because all eigenvalues of $\mathcal{L}_{5}$ have positive real part under Assumption 1 (see the work of Li et al. [31]).

Problem 2 (Output feedback-based semi-global leaderfollowing output consensus problem)

Consider a multi-agent system consisting of leader (1) and followers (2). Assume that Assumptions 1-5 hold. Let $\hat{x}=\left[\hat{x}_{1}^{\mathrm{T}}, \cdots, \hat{x}_{N}^{\mathrm{T}}\right]^{\mathrm{T}}, \hat{w}=\left[\hat{w}_{1}^{\mathrm{T}}, \cdots, \hat{w}_{N}^{\mathrm{T}}\right]^{\mathrm{T}}$. For a priori given bounded sets $\mathcal{X}_{0} \subset \mathbb{R}^{n}, \mathcal{V}_{0} \subset \mathbb{R}^{q}, \mathcal{W}_{0} \subset \mathbb{R}^{s}, \hat{\mathcal{X}}_{0} \subset$ $\mathbb{R}^{n}$ and $\hat{\mathcal{W}}_{0} \subset \mathbb{R}^{N s}$, construct an output feedback consensus protocol $u_{i}=f_{i}\left(\hat{x}_{i}, v_{i}, \hat{w}_{i}\right)$ for each follower, based on the distributed state observers (6)-(8), such that for $\left[x^{\mathrm{T}}(0), v^{\mathrm{T}}(0), w^{\mathrm{T}}(0), \hat{x}^{\mathrm{T}}(0), \hat{w}^{\mathrm{T}}(0)\right]^{\mathrm{T}} \in \mathcal{X}_{0} \times \mathcal{V}_{0} \times \mathcal{W}_{0} \times \hat{\mathcal{X}}_{0} \times$ $\hat{\mathcal{W}}_{0}$, the leader-following output consensus is achieved, that is, for any $i \in \mathcal{F}$,

$$
\lim _{t \rightarrow \infty} e_{i}=0
$$

\section{Output consensus over directed topologies}

In this section, we will propose two consensus protocols, the state feedback type and the output feedback type, to solve the semi-global leader-following output consensus problems defined in Section 2. For each $i=1,2, \cdots, N$, let $P_{i}(\epsilon)$ be the solution of the parametric ARE in (4). According to Lemma $1, P_{i}(\epsilon)$ is unique and positive definite, and it satisfies $\lim _{\epsilon \rightarrow 0} P_{i}(\epsilon)=0$. For notation convenience, we denote $P_{i}:=P_{i}(\epsilon)$ hereafter.

\subsection{Semi-global output consensus via state feedback}

In this section, based on the distributed leader state observer (5), the following state feedback consensus protocol is constructed to solve Problem 1:

$$
\begin{aligned}
u_{i}= & -\frac{1}{\epsilon^{2}} \bar{T}_{i}^{-1}\left(B_{i}^{\mathrm{T}} P_{i}\left(x_{i}-\Pi_{i} \eta_{i}\right)+\left(v_{i}-\Gamma_{i} \eta_{i}\right)\right) \\
& +\bar{T}_{i}^{-1} \Gamma_{i} S \eta_{i}+v_{i},
\end{aligned}
$$

where $P_{i}$ is the solution of (4) with $\gamma=2, \Pi_{i}$ and $\Gamma_{i}$ are a pair solution of the regulator Eq. (3), $\eta_{i}$ is the state of the observer (5). The first three terms of (9) follows from the fact that if $\lim _{t \rightarrow \infty} e_{i}=0$, then $\lim _{t \rightarrow \infty}\left(x_{i}-\Pi_{i} w\right)=0$, $\lim _{t \rightarrow \infty}\left(v_{i}-\Gamma_{i} w\right)=0$ and $\lim _{t \rightarrow \infty}\left(u_{i}-\Gamma_{i} S w\right)=0 . v_{i}$ of (9) is to make up for the term $-\bar{T}_{i} v_{i}$ of (2). The distributed control law (9) is a combination of a purely decentralized control law and a distributed observer.

Theorem 1 Consider a multi-agent system consisting of leader (1) and followers (2). Assume that Assumptions 13 and 5 hold. The state feedback consensus protocols (9) solve Problem 1. That is, for a priori given bounded sets $\mathcal{X}_{0}, \mathcal{V}_{0}, \mathcal{W}_{0}$ and $\mathcal{Z}_{0}$, there exists an $\epsilon^{*} \in(0,1]$ such that for each $\epsilon \in\left(0, \epsilon^{*}\right]$ and for all $\left[x^{\mathrm{T}}(0), v^{\mathrm{T}}(0), w^{\mathrm{T}}(0), \eta^{\mathrm{T}}(0)\right]^{\mathrm{T}} \in$ $\mathcal{X}_{0} \times \mathcal{V}_{0} \times \mathcal{W}_{0} \times \mathcal{Z}_{0}$, the output consensus error satisfies $\lim _{t \rightarrow \infty} e_{i}=0$.

Proof Denote the estimation error by $\tilde{\eta}_{i}=\eta_{i}-w$, and $\tilde{\eta}=\left[\tilde{\eta}_{1}^{\mathrm{T}}, \eta_{2}^{\mathrm{T}}, \cdots, \tilde{\eta}_{N}^{\mathrm{T}}\right]^{\mathrm{T}}$. Followed by (5), $\tilde{\eta}$ is determined by the following equation:

$$
\dot{\tilde{\eta}}=\left(I_{N} \otimes S-\mu_{1} \mathcal{L}_{f f} \otimes I_{n}\right) \tilde{\eta} .
$$

Since $I_{N} \otimes S-\mu_{1} \mathcal{L}_{f f} \otimes I_{n}$ is Hurwitz, it follows that $\lim _{t \rightarrow \infty} \tilde{\eta}_{i}=0$. Denote $\tilde{x}_{i}=x_{i}-\Pi_{i} w$, then we have

$$
\begin{aligned}
\dot{\tilde{x}}_{i} & =\dot{x}_{i}-\Pi_{i} \dot{w} \\
& =A_{i} x_{i}+B_{i} \sigma_{p}\left(v_{i}\right)+W_{i} w-\Pi_{i} S w \\
& =A_{i} \tilde{x}_{i}+B_{i} \sigma_{p}\left(v_{i}\right)-B_{i} \Gamma_{i} w,
\end{aligned}
$$

where the last equality holds is due to the first equation of (3) (Assumption 2). Let $\tilde{x}=\left[\tilde{x}_{1}^{\mathrm{T}}, \cdots, \tilde{x}_{N}^{\mathrm{T}}\right]^{\mathrm{T}}, v=$ $\left[v_{1}^{\mathrm{T}}, \cdots, v_{N}^{\mathrm{T}}\right]^{\mathrm{T}}, \bar{w}=\mathbf{1}_{N} \otimes w, \tilde{\eta}=\left[\tilde{\eta}_{1}^{\mathrm{T}}, \cdots, \tilde{\eta}_{N}^{\mathrm{T}}\right]^{\mathrm{T}}$, $A=\operatorname{diag}\left\{A_{1}, \cdots, A_{N}\right\}, B=\operatorname{diag}\left\{B_{1}, \cdots, B_{N}\right\}$, $P=\operatorname{diag}\left\{P_{1}, \cdots, P_{N}\right\}, \Pi=\operatorname{diag}\left\{\Pi_{1}, \cdots, \Pi_{N}\right\}, \Gamma=$ 
$\operatorname{diag}\left\{\Gamma_{1}, \cdots, \Gamma_{N}\right\}$. Then the compact form of (10) can be written as

$$
\dot{\tilde{x}}=A \tilde{x}+B \sigma_{p}(v)-B \Gamma \bar{w},
$$

and $v$ follows the dynamic equation

$\dot{v}=\sigma_{r}\left(-\frac{1}{\epsilon^{2}} B^{\mathrm{T}} P(x-\Pi \eta)-\frac{1}{\epsilon^{2}}(v-\Gamma \eta)+\Gamma\left(I_{N} \otimes S\right) \eta\right)$.

Define the Lyapunov function candidate

$$
V_{1}=\tilde{x}^{\mathrm{T}} P \tilde{x}+\left(B^{\mathrm{T}} P \tilde{x}+v-\Gamma \bar{w}\right)^{\mathrm{T}}\left(B^{\mathrm{T}} P \tilde{x}+v-\Gamma \bar{w}\right) .
$$

Notice that $V_{1}$ is positive definite.

According to Assumption 5, we have

$$
\left\|\Gamma_{i} w\right\|_{T, \infty}<p, \quad\left\|\Gamma_{i} S w\right\|_{T, \infty}<r .
$$

Recall that $\lim _{t \rightarrow \infty} \tilde{\eta}_{i}=0$. For simplicity, we assume

$$
\begin{aligned}
\left\|B_{i}^{\mathrm{T}} P_{i} \Pi_{i} \tilde{\eta}_{i}\right\|_{T, \infty} & \leq \epsilon^{2} \frac{\delta_{r}}{6} \\
\left\|\Gamma_{i} \tilde{\eta}_{i}\right\|_{T, \infty} & \leq \epsilon^{2} \frac{\delta_{r}}{6} \\
\left\|\Gamma_{i} S \tilde{\eta}_{i}\right\|_{T, \infty} & \leq \frac{\delta_{r}}{6}
\end{aligned}
$$

for all $\epsilon \in(0,1]$ and all initial conditions of $\tilde{\eta}_{i}(0)$. For any $\left[x^{\mathrm{T}}(0), v^{\mathrm{T}}(0), w^{\mathrm{T}}(0), \eta^{\mathrm{T}}(0)\right]^{\mathrm{T}} \in \mathcal{X}_{0} \times \mathcal{V}_{0} \times \mathcal{W}_{0} \times \mathcal{Z}_{0}, \tilde{x}(T)$ and $v(T)$ belong to bounded sets $\tilde{\mathcal{X}}_{T}$ and $\mathcal{V}_{T}$, respectively, independent of $\epsilon$, since they are determined by linear differentiate equations with bounded inputs. According to Remark 1, $w(t)$ is bounded. Therefore, there exists a bounded set $\mathcal{W}_{T}$ such that $w(T) \in \mathcal{W}_{T}$.

Let $c_{1}>0$ be a constant such that

$$
\sup _{\left.\epsilon \in(0,1], \tilde{x}^{\mathrm{T}}(T), \nu^{\mathrm{T}}(T), w^{\mathrm{T}}(T)\right]^{\mathrm{T}} \in \tilde{\mathcal{X}}_{T} \times \mathcal{V}_{T} \times \mathcal{W}_{T}} V_{1} \leq c_{1} .
$$

Such a $c_{1}$ exists because $\mathcal{X}_{T}, \mathcal{V}_{T}$ and $\mathcal{W}_{T}$ are bounded, and $\lim _{\epsilon \rightarrow 0} P=0$. Define $L_{V_{1}}\left(c_{1}\right):=\left\{\left[\tilde{x}^{\mathrm{T}}, v^{\mathrm{T}}, \bar{w}^{\mathrm{T}}\right]^{\mathrm{T}} \in\right.$ $\left.\mathbb{R}^{n+q+N s}: V_{1} \leq c_{1}\right\}$. Let $\epsilon^{*} \in(0,1]$ be such that, for all $\epsilon \in\left(0, \epsilon^{*}\right],\left[\tilde{x}^{\mathrm{T}}, v^{\mathrm{T}}, \bar{w}^{\mathrm{T}}\right]^{\mathrm{T}} \in L_{V_{1}}\left(c_{1}\right)$ implies that

$$
\begin{aligned}
\left\|B_{i}^{\mathrm{T}} P_{i} A_{i} \tilde{x}_{i}\right\|_{T, \infty} & \leq \frac{\delta_{r}}{6}, \\
\left\|B_{i}^{\mathrm{T}} P_{i} B_{i} \sigma_{p}\left(v_{i}\right)\right\|_{T, \infty} & \leq \frac{\delta_{r}}{6}, \\
\left\|B_{i}^{\mathrm{T}} P_{i} B_{i} \Gamma_{i} w\right\|_{T, \infty} & \leq \frac{\delta_{r}}{6} .
\end{aligned}
$$

The existence of such an $\epsilon^{*}$ is due to the fact that $\lim _{\epsilon \rightarrow 0} P_{i}=0$.

The derivative of $V_{1}$ along the trajectories (1), (11) and (12) inside $L_{V_{1}}\left(c_{1}\right)$ follows

$$
\begin{aligned}
\dot{V}= & \dot{\tilde{x}}^{\mathrm{T}} P \tilde{x}+\tilde{x}^{\mathrm{T}} P \dot{\tilde{x}}+2\left(B^{\mathrm{T}} P \tilde{x}+s-\Gamma \bar{w}\right)^{\mathrm{T}}\left(B^{\mathrm{T}} P \dot{\tilde{x}}+\dot{s}-\Gamma \dot{\bar{w}}\right) \\
= & \left(\tilde{x}^{\mathrm{T}} A^{\mathrm{T}}+\sigma_{p}^{\mathrm{T}}(s) B^{\mathrm{T}}-\bar{w}^{\mathrm{T}} \Gamma^{\mathrm{T}} B^{\mathrm{T}}\right) P \tilde{x} \\
& +\tilde{x}^{\mathrm{T}} P\left(A \tilde{x}+B \sigma_{p}(s)-B \Gamma \bar{w}\right) \\
& +2\left(B^{\mathrm{T}} P \tilde{x}+s-\Gamma \bar{w}\right)^{\mathrm{T}}\left(B^{\mathrm{T}} P\left(A \tilde{x}+B \sigma_{p}(s)-B \Gamma \bar{w}\right)\right. \\
& +\sigma_{r}\left[-\frac{1}{\epsilon^{2}} B^{\mathrm{T}} P(x-\Pi \eta)-\frac{1}{\epsilon^{2}}(s-\Gamma \eta)+\Gamma\left(I_{N} \otimes S\right) \eta\right] \\
& \left.-\Gamma\left(I_{N} \otimes S\right) \bar{w}\right) \\
= & \tilde{x}^{\mathrm{T}}\left(-\epsilon I_{n}+2 P B B^{\mathrm{T}} P\right) \tilde{x}+2 \tilde{x}^{v} P B \sigma_{p}(s)-2 \tilde{x}^{\mathrm{T}} P B \Gamma \bar{w} \\
& +2\left(B^{\mathrm{T}} P \tilde{x}+s-\Gamma \bar{w}\right)^{\mathrm{T}} \\
& \times\left(\sigma_{r}\left[-\frac{1}{\epsilon^{2}} B^{\mathrm{T}} P \tilde{x}-\frac{1}{\epsilon^{2}}(s-\Gamma \bar{w})+\Gamma\left(I_{N} \otimes S\right) \bar{w}+\varsigma\right]\right. \\
& \left.-\Gamma\left(I_{N} \otimes S\right) \bar{w}+\Theta\right) \\
= & -\epsilon \tilde{x}^{\mathrm{T}} \tilde{x}+2 \tilde{x}^{\mathrm{T}} P B\left(B^{\mathrm{T}} P \tilde{x}+\sigma_{p}(s)-\Gamma \bar{w}\right) \\
& +2\left(B^{\mathrm{T}} P \tilde{x}+s-\Gamma \bar{w}\right)^{\mathrm{T}} \\
& \times\left(\sigma_{r}\left[-\frac{1}{\epsilon^{2}} B^{\mathrm{T}} P \tilde{x}-\frac{1}{\epsilon^{2}}(s-\Gamma \bar{w})+\Gamma\left(I_{N} \otimes S\right) \bar{w}+\varsigma\right]\right. \\
& \left.-\Gamma\left(I_{N} \otimes S\right) \bar{w}+\Theta\right) \\
& -\epsilon \tilde{x}^{\mathrm{T}} \tilde{x}+\sum_{i=1}^{N} 2 \tilde{x}_{i}^{\mathrm{T}} P_{i} B_{i}\left(B_{i}^{\mathrm{T}} P_{i} \tilde{x}_{i}+\sigma_{p}\left(s_{i}\right)-\Gamma_{i} w\right) \\
& +\sum_{i=1}^{N} 2\left(B_{i}^{\mathrm{T}} P_{i} \tilde{x}_{i}+s_{i}-\Gamma_{i} w\right)^{\mathrm{T}} \\
& +\left(\sigma_{r}\left[-\frac{1}{\epsilon^{2}} B_{i}^{\mathrm{T}} P_{i} \tilde{x}_{i}-\frac{1}{\epsilon^{2}}\left(s_{i}-\Gamma_{i} w\right)+\Gamma_{i} S w+\theta_{i}\right)\right. \\
& \\
& \\
&
\end{aligned}
$$

with $\varsigma=\frac{1}{\epsilon^{2}} B^{\mathrm{T}} P \Pi \tilde{\eta}+\frac{1}{\epsilon^{2}} \Gamma \tilde{\eta}+\Gamma\left(I_{N} \otimes S\right) \tilde{\eta}, \Theta=B^{\mathrm{T}} P(A \tilde{x}+$ $\left.B \sigma_{p}(v)-B \Gamma \bar{w}\right), \varsigma_{i}=\frac{1}{\epsilon^{2}} B_{i}^{\mathrm{T}} P_{i} \Pi_{i} \tilde{\eta}_{i}+\frac{1}{\epsilon^{2}} \Gamma_{i} \tilde{\eta}_{i}+\Gamma_{i} S \tilde{\eta}_{i}$, and 
$\theta_{i}=B_{i}^{\mathrm{T}} P_{i}\left(A_{i} \tilde{x}_{i}+B_{i} \sigma_{p}\left(v_{i}\right)-B_{i} \Gamma_{i} w\right)$. Denote $\phi_{i}=-B_{i}^{\mathrm{T}} P_{i} \tilde{x}_{i}$, the derivative of $V_{1}$ can be rewritten as

$$
\begin{aligned}
\dot{V}_{1}= & -\epsilon \tilde{x} \mathrm{~T} \tilde{x}-\sum_{i=1}^{N} 2 \phi_{i}^{\mathrm{T}}\left(\sigma_{p}\left(v_{i}\right)-\Gamma_{i} w-\phi_{i}\right) \\
& +\sum_{i=1}^{N} 2\left(v_{i}-\Gamma_{i} w-\phi_{i}\right)^{\mathrm{T}} \\
& \times\left(\sigma_{r}\left[-\frac{1}{\epsilon^{2}}\left(v_{i}-\Gamma_{i} w-\phi_{i}\right)+\Gamma_{i} S w+\varsigma_{i}\right]\right. \\
& \left.-\Gamma_{i} S w+\theta_{i}\right) .
\end{aligned}
$$

According to (13) and (14), we have

$\left\|S_{i}\right\| \leq\left\|\frac{1}{\epsilon^{2}} B_{i}^{\mathrm{T}} P_{i} \Pi_{i} \tilde{\eta}_{i}\right\|+\left\|\frac{1}{\epsilon^{2}} \Gamma_{i} \tilde{\eta}_{i}\right\|+\left\|\Gamma_{i} S \tilde{\eta}_{i}\right\| \leq \frac{\delta_{r}}{2}$,

$\left\|\theta_{i}\right\| \leq\left\|B_{i}^{\mathrm{T}} P_{i} A_{i} \tilde{x}_{i}\right\|+\left\|B_{i}^{\mathrm{T}} P_{i} B_{i} \delta_{p}\left(v_{i}\right)\right\|+\left\|B_{i}^{\mathrm{T}} P_{i} B_{i} \Gamma_{i} w\right\| \leq \frac{\delta_{r}}{2}$.

(i) We first consider the case that

$\left\|-\frac{1}{\epsilon^{2}}\left(v_{i}-\Gamma_{i} w-\phi_{i}\right)+\Gamma_{i} S w+\varsigma_{i}\right\| \leq r$,

that is, $\left\|v_{i}-\Gamma_{i} w-\phi_{i}\right\| \leq \epsilon^{2} \frac{\delta_{r}}{2}$,

which implies $\left\|v_{i}\right\| \leq \epsilon^{2} \frac{\delta_{r}}{2}+\left\|\Gamma_{i} w\right\|+\left\|\phi_{i}\right\|$.

Choosing $\epsilon \leq \min \left\{\max \left\{\epsilon: \epsilon \in(0,1],\left\|\phi_{i}\right\| \leq \delta_{p}-\right.\right.$ $\left.\left.\left\|\Gamma_{i} w\right\|\right\}, \sqrt{\frac{2\left(p-\left\|\Gamma_{i} w\right\|-\left\|\phi_{i}\right\|\right)}{\delta_{r}}}\right\}$. Such an $\epsilon$ exists because $\left\|\Gamma_{i} w\right\|<p$ and $\lim _{\epsilon \rightarrow 0}\left\|\phi_{i}\right\|=\lim _{\epsilon \rightarrow 0}\left\|-B_{i}^{\mathrm{T}} P_{i} \tilde{x}_{i}\right\|=0$. Then, we have $\left\|v_{i}\right\| \leq p$, which means $\sigma_{p}\left(v_{i}\right)=v_{i}$. It follows that

$$
\begin{aligned}
& \sigma_{r}\left[-\frac{1}{\epsilon^{2}}\left(v_{i}-\Gamma_{i} w-\phi_{i}\right)+\Gamma_{i} S w+\varsigma_{i}\right]-\Gamma_{i} S w+\theta_{i} \\
& =-\frac{1}{\epsilon^{2}}\left(v_{i}-\Gamma_{i} w-\phi_{i}\right)+\varsigma_{i}+\theta_{i}
\end{aligned}
$$

and

$$
2 \phi_{i}^{\mathrm{T}}\left(\sigma_{p}\left(v_{i}\right)-\Gamma_{i} w-\phi_{i}\right)=2 \phi_{i}^{\mathrm{T}}\left(v_{i}-\Gamma_{i} w-\phi_{i}\right) .
$$

Taking (17) and (18) into (16) gives

$$
\begin{aligned}
\dot{V}_{1}= & -\epsilon \tilde{x}^{\mathrm{T}} \tilde{x}-\sum_{i=1}^{N} 2 \phi_{i}^{\mathrm{T}}\left(v_{i}-\Gamma_{i} w-\phi_{i}\right) \\
& +\sum_{i=1}^{N} 2\left(v_{i}-\Gamma_{i} w-\phi_{i}\right)^{\mathrm{T}} \\
& \times\left(-\frac{1}{\epsilon^{2}}\left(v_{i}-\Gamma_{i} w-\phi_{i}\right)+\varsigma_{i}+\theta_{i}\right) \\
\leq & -\epsilon \tilde{x}^{\mathrm{T}} \tilde{x}-2 \sum_{i=1}^{N}\left(\frac{1}{\epsilon^{2}}\left\|v_{i}-\Gamma_{i} w-\phi_{i}\right\|-\| \zeta_{i}\right. \\
& \left.+\theta_{i}\|+\| \phi_{i} \|\right)\left\|v_{i}-\Gamma_{i} w-\phi_{i}\right\| .
\end{aligned}
$$

There exists an $\epsilon_{1}^{*} \in(0,1]$ such that for any $\epsilon \in\left(0, \epsilon_{1}^{*}\right], \frac{1}{\epsilon^{2}}\left\|\nu_{i}-\Gamma_{i} w-\phi_{i}\right\|-\left\|\varsigma_{i}+\theta_{i}\right\|+\left\|\phi_{i}\right\|>0$, because $\frac{1}{\epsilon^{2}} \rightarrow \infty$ as $\epsilon \rightarrow 0,\left\|\phi_{i}\right\| \rightarrow 0$ as $\epsilon \rightarrow 0$, and $\left\|s_{i}+\theta_{i}\right\| \leq \delta_{r}$. Thus, we have

$$
\dot{V}_{1}<0, \forall\left[\tilde{x}^{\mathrm{T}}, v^{\mathrm{T}}, \bar{w}^{\mathrm{T}}\right]^{\mathrm{T}} \in L_{V_{1}}\left(c_{1}\right) \backslash\{0\} .
$$

(ii) Next, we consider the case that

$-\frac{1}{\epsilon^{2}}\left(v_{i}-\Gamma_{i} w-\phi_{i}\right)+\Gamma_{i} S w+\varsigma_{i}<-r$, that is

$v_{i}-\Gamma_{i} w-\phi_{i}>\epsilon^{2}\left(r+\Gamma_{i} S w+\varsigma_{i}\right)>0$.

(a) if $v_{i}-\Gamma_{i} w>0$ and $\phi_{i}>0$, then $\sigma_{p}\left(v_{i}\right)-\Gamma_{i} w \geq 0$. It follows that (16) can be rewritten as

$$
\begin{aligned}
\dot{V}_{1} \leq & -\epsilon \tilde{x}^{\mathrm{T}} \tilde{x}-2 \sum_{i=1}^{N}\left\|\phi_{i}\right\|\left(v_{i}-\Gamma_{i} w-\phi_{i}\right) \\
& +2 \sum_{i=1}^{N}\left(-r-\Gamma_{i} S w+\left\|\theta_{i}\right\|\right)\left(v_{i}\right. \\
& \left.-\Gamma_{i} w-\phi_{i}\right) \\
= & -\epsilon \tilde{x}^{\mathrm{T}} \tilde{x}-2 \sum_{i=1}^{N}\left(\left\|\phi_{i}\right\|+r+\Gamma_{i} S w\right. \\
& \left.-\left\|\theta_{i}\right\|\right)\left(v_{i}-\Gamma_{i} w-\phi_{i}\right) .
\end{aligned}
$$

Since $r-\left\|\Gamma_{i} S w\right\|<\delta_{r}$ and $\left\|\theta_{i}\right\| \leq \frac{\delta_{r}}{2}$, there exists an $\epsilon_{2}^{*} \in(0,1]$ such that for any $\epsilon \in\left(0, \epsilon_{2}^{*}\right],\left\|\phi_{i}\right\|+r+\Gamma_{i} S w-\left\|\theta_{i}\right\|>0$. Thus, it follows that (19) holds.

(b) if $v_{i}-\Gamma_{i} w>0$ and $\phi_{i}<0$, then

$$
\begin{gathered}
\left(v_{i}-\Gamma_{i} w-\phi_{i}\right)^{\mathrm{T}}\left(-r-\Gamma_{i} S w+\theta_{i}\right)<0 \text { and } \\
-\phi_{i}^{\mathrm{T}}\left(\sigma_{p}\left(v_{i}\right)-\Gamma_{i} w-\phi_{i}\right)>0 . \text { Then, we have } \\
\dot{V}_{1}=-\epsilon \tilde{x}^{\mathrm{T}} \tilde{x}-2 \sum_{i=1}^{N} \phi_{i}^{\mathrm{T}}\left(\sigma_{p}\left(v_{i}\right)-\Gamma_{i} w-\phi_{i}\right) \\
\quad+2 \sum_{i=1}^{N}\left(v_{i}-\Gamma_{i} w-\phi_{i}\right)^{\mathrm{T}}\left(-r-\Gamma_{i} S w+\theta_{i}\right) \\
\leq-\epsilon \tilde{x}^{\mathrm{T}} \tilde{x}+2 \sum_{i=1}^{N}\left(-r-\Gamma_{i} S w+\theta_{i}+\left\|\phi_{i}\right\|\right) \\
\quad\left(v_{i}-\Gamma_{i} w-\phi_{i}\right) .
\end{gathered}
$$

Similarly, there exists an $\epsilon_{3}^{*} \in(0,1]$ such that for any $\epsilon \in\left(0, \epsilon_{3}^{*}\right],-r-\Gamma_{i} S w+\theta_{i}+\left\|\phi_{i}\right\|<0$. Since $v_{i}-\Gamma_{i} w-\phi_{i}>0$, we have $\dot{V}_{1} \leq-\epsilon \tilde{x}^{\mathrm{T}} \tilde{x}$, which means (19) holds.

(c) if $v_{i}-\Gamma_{i} w<0$ and $\phi_{i}<0$, then $v_{i}<\Gamma_{i} w<p$. It is easy to verify that (20) holds.

(iii) Similarly, we can show that (19) holds for the case that $-\frac{1}{\epsilon^{2}}\left(v_{i}-\Gamma_{i} w-\phi_{i}\right)+\Gamma_{i} S w+\varsigma_{i}>r$. 
In conclusion, we have shown that, for all $\epsilon \in\left(0, \epsilon^{*}\right]$, $\epsilon^{*}=\min \left\{\epsilon_{1}^{*}, \epsilon_{2}^{*}, \epsilon_{3}^{*}\right\}$,

$$
\dot{V}_{1}<0, \forall\left[\tilde{x}^{\mathrm{T}}, v^{\mathrm{T}}, \bar{w}^{\mathrm{T}}\right]^{\mathrm{T}} \in L_{V_{1}}\left(c_{1}\right) \backslash\{0\} .
$$

Hence, we have $\lim _{t \rightarrow \infty} \tilde{x}_{i}=\lim _{t \rightarrow \infty}\left(x_{i}-\Pi_{i} w\right)=0$ and $\lim _{t \rightarrow \infty}\left(v_{i}-\Gamma_{i} w\right)=0$, which implies that

$\lim _{t \rightarrow \infty} e_{i}=\lim _{t \rightarrow \infty}\left(C_{i} x_{i}+Q w\right)=\lim _{t \rightarrow \infty}\left(C_{i}\left(\tilde{x}_{i}+\Pi_{i} w\right)+Q w\right)=0$.

The above facts complete the proof.

\subsection{Semi-global output consensus via output feedback}

In this section, we construct the following output feedback consensus protocol based on the distributed observers (6)-(8):

$$
\begin{aligned}
u_{i}= & -\frac{1}{\epsilon^{2}} \bar{T}_{i}^{-1}\left(B_{i}^{\mathrm{T}} P_{i}\left(\hat{x}_{i}-\Pi_{i} \hat{w}_{i}\right)+\left(v_{i}-\Gamma_{i} \hat{w}_{i}\right)\right) \\
& +\bar{T}_{i}^{-1} \Gamma_{i} S \hat{w}_{i}+v_{i},
\end{aligned}
$$

where $P_{i}$ is the solution of (4) with $\gamma=1, \Pi_{i}$ and $\Gamma_{i}$ are a pair solution of the regulator Eq. (3), $\hat{x}_{i}$ and $\hat{w}_{i}$ are the states of the distributed observers (6)-(8). The idea of designing control law (21) is similar to the design of control law (9).

Theorem 2 Consider a multi-agent system consisting of leader (1) and followers (2). Assume that Assumptions 1-5 hold. The output feedback consensus protocols (21) solve Problem 2. That is, for a priori given bounded sets $\mathcal{X}_{0}, \mathcal{V}_{0}, \mathcal{W}_{0}, \hat{\mathcal{X}}_{0}$ and $\hat{\mathcal{W}}_{0}$, there exists an $\epsilon^{*} \in(0,1]$ such that for each $\epsilon \in\left(0, \epsilon^{*}\right]$ and for all $\left[x^{\mathrm{T}}(0), v^{\mathrm{T}}(0), w^{\mathrm{T}}(0), \hat{x}^{\mathrm{T}}(0), \hat{w}^{\mathrm{T}}(0)\right]^{\mathrm{T}} \in \mathcal{X}_{0} \times \mathcal{V}_{0} \times \mathcal{W}_{0} \times \hat{\mathcal{X}}_{0} \times$ $\hat{\mathcal{W}}_{0}$, the output consensus error satisfies $\lim _{t \rightarrow \infty} e_{i}=0$.

Proof Firstly, we show that $\lim _{t \rightarrow \infty}\left(\hat{w}_{i}-w\right)=0$. Denote $\breve{w}_{i}=\hat{w}_{i}-w, i=1,2, \cdots, N, \breve{w}_{i n}=\left[\breve{w}_{1}^{\mathrm{T}}, \cdots, \breve{w}_{l}^{\mathrm{T}}\right]^{\mathrm{T}}$, and $\breve{w}_{u n}=\left[\breve{w}_{l+1}^{\mathrm{T}}, \cdots, \breve{w}_{N}^{\mathrm{T}}\right]^{\mathrm{T}}$. By the observers (6) and (7), we have

$$
\begin{aligned}
\dot{\breve{w}}_{i n} & =D_{1} \breve{w}_{i n}, \\
\dot{\breve{w}}_{u n} & =D_{2} \breve{w}_{u n}+E \breve{w}_{i n},
\end{aligned}
$$

where $D_{1}=I_{l} \otimes\left(S+L_{S, i} Q\right), D_{2}=I_{N-l} \otimes S-\mu_{2} \mathcal{L}_{5} \otimes I_{s}$ and $E=-\mu_{2}\left(\mathcal{L}_{4} \otimes I_{s}\right)$. Since both $D_{1}$ and $D_{2}$ are Hurwitz, it is obvious that $\lim _{t \rightarrow \infty} \breve{w}_{i}=0$.

Denote $\breve{x}_{i}=\hat{x}_{i}-x_{i}$, then it follows from (8) that

$$
\breve{x}_{i}=\left(A_{i}+L_{A, i} C_{i}\right) \breve{x}_{i}-B_{i} \sigma\left(v_{i}\right)+W_{i} \breve{w}_{i} .
$$

Let $H_{i}=A_{i}+L_{A, i} C_{i}$. Since $H_{i}$ is Hurwitz, for any positive definite matrix $N_{i}$, there exists a positive definite matrix $M_{i}$ that satisfies

$$
H_{i}^{\mathrm{T}} M_{i}+M_{i} H_{i}=-N_{i} .
$$

(10) is independent of the control law, so it also holds.
For each follower, we define a Lyapunov function candidate

$$
\begin{aligned}
& V_{2, i}=\tilde{x}_{i}^{\mathrm{T}} P_{i} \tilde{x}_{i}+\lambda_{\max }\left(P_{i}\right) \breve{x}_{i}^{\mathrm{T}} M_{i} \breve{x}_{i} \\
& \\
&+\left(B_{i}^{\mathrm{T}} P_{i} \tilde{x}_{i}+v_{i}-\Gamma_{i} w+B_{i}^{\mathrm{T}} P_{i} \breve{x}_{i}\right)^{\mathrm{T}} \\
& \times\left(B_{i}^{\mathrm{T}} P_{i} \tilde{x}_{i}+v_{i}-\Gamma_{i} w+B_{i}^{\mathrm{T}} P_{i} \breve{x}_{i}\right) .
\end{aligned}
$$

Let $c_{2, i}$ be a constant scalar such that

$$
\sup _{\substack{\epsilon \in(0,1],\left[x_{i}^{\mathrm{T}}(0), v_{i}^{\mathrm{T}}(0), w^{\mathrm{T}}(0), \hat{x}_{i}^{\mathrm{T}}(0), \hat{w}_{i}^{\mathrm{T}}(0)\right]^{\mathrm{T}} \\ \in \mathcal{X}_{0} \times \mathcal{V}_{0} \times \mathcal{W}_{0} \times \mathcal{\mathcal { X }}_{0} \times \mathcal{\mathcal { W }}_{0}}} V_{2, i} \leq c_{2, i} .
$$

Such a $c_{2, i}$ exists because sets $\mathcal{X}_{0}, \mathcal{V}_{0}, \mathcal{W}_{0}, \hat{\mathcal{X}}_{0}$ and $\hat{\mathcal{W}}_{0}$ are bounded, and $\lim _{\epsilon \rightarrow 0} P_{i}=0$. Define $L_{V_{2, i}}:=$ $\left\{\left(x_{i}, v_{i}, w, \hat{x}_{i}, \hat{w}_{i}\right) \in \mathbb{R}^{2 n_{i}+2 s+q_{i}}: V_{2, i} \leq c_{2, i}\right\}$. Let $\epsilon_{i}^{*} \in(0,1]$ be such that, for all $\epsilon \in\left(0, \epsilon_{i}^{*}\right],\left(x_{i}, v_{i}, w, \hat{x}_{i}, \hat{w}_{i}\right) \in L_{V_{2, i}}$ implies that

$$
\begin{array}{r}
\left\|B_{i}^{\mathrm{T}} P_{i}\left(A_{i} \tilde{x}_{i}+B_{i} \sigma_{p}\left(v_{i}\right)-B_{i} \Gamma_{i} w\right)\right\| \leq \frac{\delta_{r}}{8}, \\
\left\|B_{i}^{\mathrm{T}} P_{i}\left(H_{i} \breve{x}_{i}+W_{i} \breve{w}_{i}-B_{i} \sigma_{p}\left(v_{i}\right)\right)\right\| \leq \frac{\delta_{r}}{8}, \\
\left\|B_{i}^{\mathrm{T}} M_{i} \breve{x}_{i}\right\| \leq \frac{\delta_{r}}{4 \lambda_{\max }\left(P_{i}\right)} .
\end{array}
$$

The existence of such an $\epsilon_{i}^{*}$ is due to $\lim _{\epsilon \rightarrow 0} P_{i}=0$.

The derivative of $V_{2, i}$ is

$$
\begin{aligned}
\dot{V}_{2, i}= & -\epsilon \tilde{x}_{i}^{\mathrm{T}} \tilde{x}_{i}+2 \tilde{x}_{i}^{\mathrm{T}} P_{i} B_{i}\left(0.5 B_{i}^{\mathrm{T}} P_{i} \tilde{x}_{i}+\sigma_{p}\left(v_{i}\right)-\Gamma_{i} w\right) \\
& -\lambda_{\max }\left(P_{i}\right) \breve{x}_{i}^{\mathrm{T}} N_{i} \breve{x}_{i} \\
& +2 \lambda_{\max } \breve{x}_{i}^{\mathrm{T}} M_{i} W_{i} \breve{w}_{i}-2 \lambda_{\max }\left(P_{i}\right) \breve{x}_{i}^{\mathrm{T}} M_{i} B_{i} \sigma_{p}\left(v_{i}\right) \\
& +2\left(v_{i}-\Gamma_{i} w+B_{i}^{\mathrm{T}} P_{i} \tilde{x}_{i}+B_{i}^{\mathrm{T}} P_{i} \breve{x}_{i}\right) \\
& \times\left(\sigma_{r}\left(-\bar{T}_{i} v_{i}+\bar{T}_{i} u_{i}\right)-\Gamma_{i} S w\right. \\
& +B_{i}^{\mathrm{T}} P_{i}\left(A_{i} \tilde{x}_{i}+B_{i} \sigma_{p}\left(v_{i}\right)-B_{i} \Gamma_{i} w\right) \\
& \left.+B_{i}^{\mathrm{T}} P_{i}\left(H_{i} \breve{x}_{i}+W_{i} \breve{w}_{i}-B_{i} \sigma_{p}\left(v_{i}\right)\right)\right) .
\end{aligned}
$$

Let

$$
\begin{array}{r}
\phi_{i}=-B_{i}^{\mathrm{T}} P_{i} \tilde{x}_{i}, \quad \beta_{i}=B_{i}^{\mathrm{T}} P_{i} \breve{x}_{i}, \quad \psi_{i}=B_{i}^{\mathrm{T}} M_{i} \breve{x}_{i}, \\
\theta_{i}=B_{i}^{\mathrm{T}} P_{i}\left(A_{i} \tilde{x}_{i}+B_{i} \sigma_{p}\left(v_{i}\right)-B_{i} \Gamma_{i} w\right), \\
\alpha_{i}=B_{i}^{\mathrm{T}} P_{i}\left(H_{i} \breve{x}_{i}+W_{i} \breve{w}_{i}-B_{i} \sigma_{p}\left(v_{i}\right)\right) .
\end{array}
$$


Then, (26) can be written as

$$
\begin{aligned}
\dot{V}_{2, i}= & -\epsilon \tilde{x}_{i}^{\mathrm{T}} \tilde{x}_{i}-\lambda_{\max }\left(P_{i}\right) \breve{x}_{i}^{\mathrm{T}} N_{i} \breve{x}_{i}+2 \lambda_{\max } \breve{x}_{i}^{\mathrm{T}} M_{i} W_{i} \breve{w}_{i} \\
& +2\left(\frac{1}{2} \phi_{i}^{2}-\phi_{i} \sigma_{p}\left(v_{i}\right)+\phi_{i} \Gamma_{i} w\right)-2 \lambda_{\max }\left(P_{i}\right) \psi_{i} \sigma_{p}\left(v_{i}\right) \\
& +2\left(v_{i}-\Gamma_{i} w-\phi_{i}+\beta_{i}\right) \\
& \times\left[\sigma _ { r } \left(-\frac{1}{\epsilon^{2}}\left(-\phi_{i}+\beta_{i}+v_{i}-\Gamma_{i} w-\Gamma_{i} \breve{w}_{i}\right)+\Gamma_{i} S w\right.\right. \\
& \left.\left.\quad+\Gamma_{i} S \breve{w}_{i}\right)-\Gamma_{i} S w-\theta_{i}-\alpha_{i}\right] .
\end{aligned}
$$

Moreover,

$$
\begin{aligned}
& 2\left(\frac{1}{2} \phi_{i}^{2}-\phi_{i} \sigma_{p}\left(v_{i}\right)+\phi_{i} \Gamma_{i} w\right)-2 \lambda_{\max }\left(P_{i}\right) \psi_{i} \sigma_{p}\left(v_{i}\right) \\
= & 2\left(-\frac{1}{4} \phi_{i}^{2}-\phi_{i}\left(\sigma_{p}\left(v_{i}\right)-\Gamma_{i} w-\phi_{i}\right)\right) \\
+ & 2\left(-\lambda_{\max }\left(P_{i}\right) \psi_{i} \sigma_{p}\left(v_{i}\right)-\frac{1}{4} \phi_{i}^{2}\right) .
\end{aligned}
$$

Since $\sigma_{p}$ is a standard saturation function, we have

$$
\left|\sigma_{p}\left(s_{1}\right)-\sigma_{p}\left(s_{2}\right)\right| \leq\left|s_{1}-s_{2}\right| .
$$

Then, in the case of $-\frac{1}{\epsilon^{2}}\left(-\phi_{i}+\beta_{i}+v_{i}-\Gamma_{i} w-\Gamma_{i} \breve{w}_{i}\right)+$ $\Gamma_{i} S w+\Gamma_{i} S \breve{w}_{i}<-r$, it follows that

$$
\begin{aligned}
& -\lambda_{\max }\left(P_{i}\right) \psi_{i} \sigma_{p}\left(v_{i}\right)-\frac{1}{4} \phi_{i}^{2} \\
= & -\lambda_{\max }\left(P_{i}\right) \psi_{i}\left(\sigma_{p}\left(v_{i}-\Gamma_{i} w-\phi_{i}+\beta_{i}\right)\right. \\
& \left.-\sigma_{p}\left(v_{i}-\Gamma_{i} w-\phi_{i}+\beta_{i}\right)+\sigma_{p}\left(v_{i}\right)\right)-\frac{1}{4} \phi_{i}^{2} \\
\leq & \frac{\delta_{r}}{4}\left\|v_{i}-\Gamma_{i} w-\phi_{i}+\beta_{i}\right\|+\lambda_{\max }\left(P_{i}\right)\left(\left|\psi_{i} \Gamma_{i} w\right|+\left|\psi_{i} \beta_{i}\right|\right) \\
& +\lambda_{\max }\left(P_{i}\right)\left|\psi_{i} \phi_{i}\right|-\frac{1}{4} \phi_{i}^{2} \\
\leq & \frac{\delta_{r}}{4}\left\|v_{i}-\Gamma_{i} w-\phi_{i}+\beta_{i}\right\|+k \lambda_{\max }\left(P_{i}\right) \psi_{i}^{2} \\
& +\lambda_{\max }\left(P_{i}\right)\left|\psi_{i} \beta_{i}\right|+\lambda_{\max }^{2}\left(P_{i}\right) \psi_{i}^{2} \\
\leq & \frac{\delta_{r}}{4}\left\|v_{i}-\Gamma_{i} w-\phi_{i}+\beta_{i}\right\|+\lambda_{\max }\left(P_{i}\right)[(k+1 \\
& \left.+\lambda_{\max }\left(P_{i}\right)\right)\left\|M_{i} B_{i} B_{i}^{\mathrm{T}} M_{i}\right\| \\
& \left.+\lambda_{\max }\left(P_{i}\right)\left\|M_{i} B_{i} B_{i}^{\mathrm{T}} P_{i}\right\|\right] \breve{x}_{i}^{\mathrm{T}} \breve{x}_{i}
\end{aligned}
$$

where we assume $\left\|\Gamma_{i} w\right\| \leq k\left\|\psi_{i}\right\|$ because $\left\|\Gamma_{i} w\right\|$ is bounded according to Assumption 5. In addition,

$$
\begin{aligned}
& -\frac{1}{4} \phi_{i}^{2}-\phi_{i}\left(\sigma_{p}\left(v_{i}\right)-\Gamma_{i} w-\phi_{i}\right) \\
= & -\frac{1}{4} \phi_{i}^{2}-\phi_{i}\left(\sigma_{p}\left(v_{i}\right)-\Gamma_{i} w-\phi_{i}+\beta_{i}\right)+\psi_{i} \beta_{i} \\
\leq & \frac{\delta_{r}}{4}\left\|v_{i}-\Gamma_{i} w-\phi_{i}+\beta_{i}\right\|+\beta_{i}^{2} \\
\leq & \frac{\delta_{r}}{4}\left\|v_{i}-\Gamma_{i} w-\phi_{i}+\beta_{i}\right\|+\left\|P_{i} B_{i} B_{i}^{\mathrm{T}} P_{i}\right\| \breve{x}_{i}^{\mathrm{T}} \breve{x}_{i} .
\end{aligned}
$$

The last term of (28) follows that

$$
\begin{aligned}
& \left(v_{i}-\Gamma_{i} w-\phi_{i}+\beta_{i}\right)\left[\sigma _ { r } \left(-\frac{1}{\epsilon^{2}}\left(-\phi_{i}+\beta_{i}+v_{i}-\Gamma_{i} w\right.\right.\right. \\
& \left.\left.\left.-\Gamma_{i} \breve{w}_{i}\right)+\Gamma_{i} S w+\Gamma_{i} S \breve{w}_{i}\right)-\Gamma_{i} S w-\theta_{i}-\alpha_{i}\right] \\
\leq & \left\|v_{i}-\Gamma_{i} w-\phi_{i}+\beta_{i}\right\|\left(-r+r-\delta_{r}+\left\|\theta_{i}\right\|_{\|} \alpha_{i} \|\right) \\
\leq & -\frac{3 \delta_{r}}{4}\left\|v_{i}-\Gamma_{i} w-\phi_{i}+\beta_{i}\right\| .
\end{aligned}
$$

Then, taking (29)-(31) into (28) gives

$$
\begin{aligned}
& \dot{V}_{2, i} \\
& \leq-\epsilon \tilde{x}_{i}^{\mathrm{T}} \tilde{x}_{i}+2 \lambda_{\max } \breve{x}_{i}^{\mathrm{T}} M_{i} W_{i} \breve{w}_{i}-\frac{\delta_{r}}{2} \| v_{i}-\Gamma_{i} w \\
& \quad-\phi_{i}+\beta_{i} \| \\
& \quad-\left(\lambda_{\max }\left(P_{i}\right)\left\|N_{i}\right\|-2 \lambda_{\max }\left(P_{i}\right)[(k+1\right. \\
& \left.\quad+\lambda_{\max }\left(P_{i}\right)\right)\left\|M_{i} B_{i} B_{i}^{\mathrm{T}} M_{i}\right\| \\
& \left.\left.\quad+\lambda_{\max }\left(P_{i}\right)\left\|M_{i} B_{i} B_{i}^{\mathrm{T}} P_{i}\right\|\right]-2\left\|P_{i} B_{i} B_{i}^{\mathrm{T}} P_{i}\right\|\right) \breve{x}_{i}^{\mathrm{T}} \breve{x}_{i} .
\end{aligned}
$$

Choose $N_{i}$ large enough such that

$$
\begin{aligned}
& \lambda_{\max }\left(P_{i}\right)\left\|N_{i}\right\|-2\left\|P_{i} B_{i} B_{i}^{\mathrm{T}} P_{i}\right\|-2 \lambda_{\max }\left(P_{i}\right)[(k+1 \\
& \left.\left.+\lambda_{\max }\left(P_{i}\right)\right)\left\|M_{i} B_{i} B_{i}^{\mathrm{T}} M_{i}\right\|+\lambda_{\max }\left(P_{i}\right)\left\|M_{i} B_{i} B_{i}^{\mathrm{T}} P_{i}\right\|\right] \\
& >0 .
\end{aligned}
$$

Hence, we have

$$
\dot{V}_{2, i}<0, \epsilon \in\left(0, \epsilon_{i}^{*}\right] \text {. }
$$

Similarly, we can show that $\dot{V}_{2, i}<0$ when $-\frac{1}{\epsilon^{2}}\left(-\phi_{i}+\beta_{i}+v_{i}-\Gamma_{i} w-\Gamma_{i} \breve{w}_{i}\right)+\Gamma_{i} S w+\Gamma_{i} S \breve{w}_{i}>r$ or $\left\|-\frac{1}{\epsilon^{2}}\left(-\phi_{i}+\beta_{i}+v_{i}-\Gamma_{i} w-\Gamma_{i} \breve{w}_{i}\right)+\Gamma_{i} S w+\Gamma_{i} S \breve{w}_{i}\right\|<$ $r$.

\section{Illustrative examples}

In this section, two examples are given to verify the effectiveness of the state feedback consensus protocol (9) and output feedback consensus protocol (21), which, respectively, solves the output consensus problem defined in

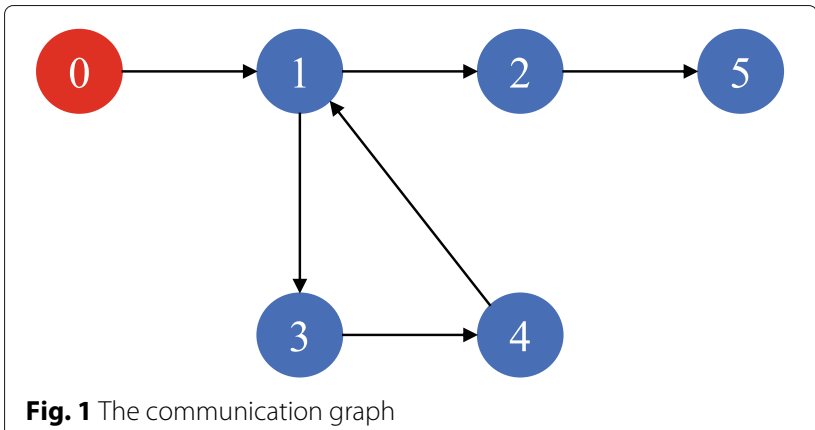



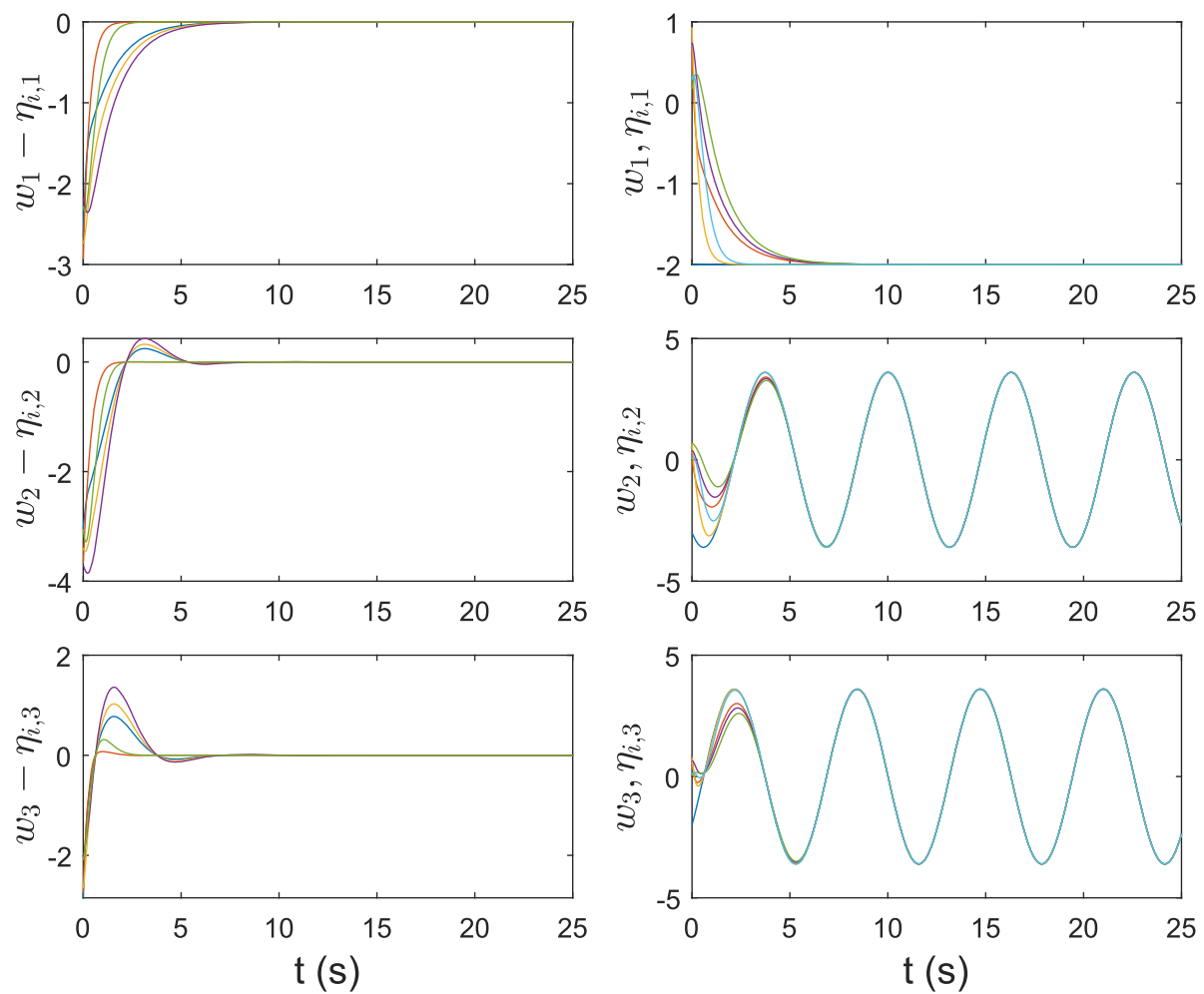

Fig. 2 (A), Estimation errors $w-\eta_{i}, i=1,2, \ldots, 5$; (B), States of the leader $w$ and states of the distributed observer (5) $\eta_{i}, i=1,2, \ldots, 5$

Problem 1 and Problem 2. The control laws have also been applied successfully to formation control in practical scenarios in [32].

The multi-agent system consists of one leader (labeled as 0 ), one informed follower (labeled as 1) and four unin- formed followers (labeled as 2, 3, 4 and 5, respectively). The communication graph $\mathcal{G}$ shown in Fig. 1 is a directed network containing a loop. It is clear that Assumption 1 is satisfied. The corresponding Laplacian matrix $\mathcal{L}, \mathcal{L}_{f f}$ and $\mathcal{L}_{5}$ are respectively
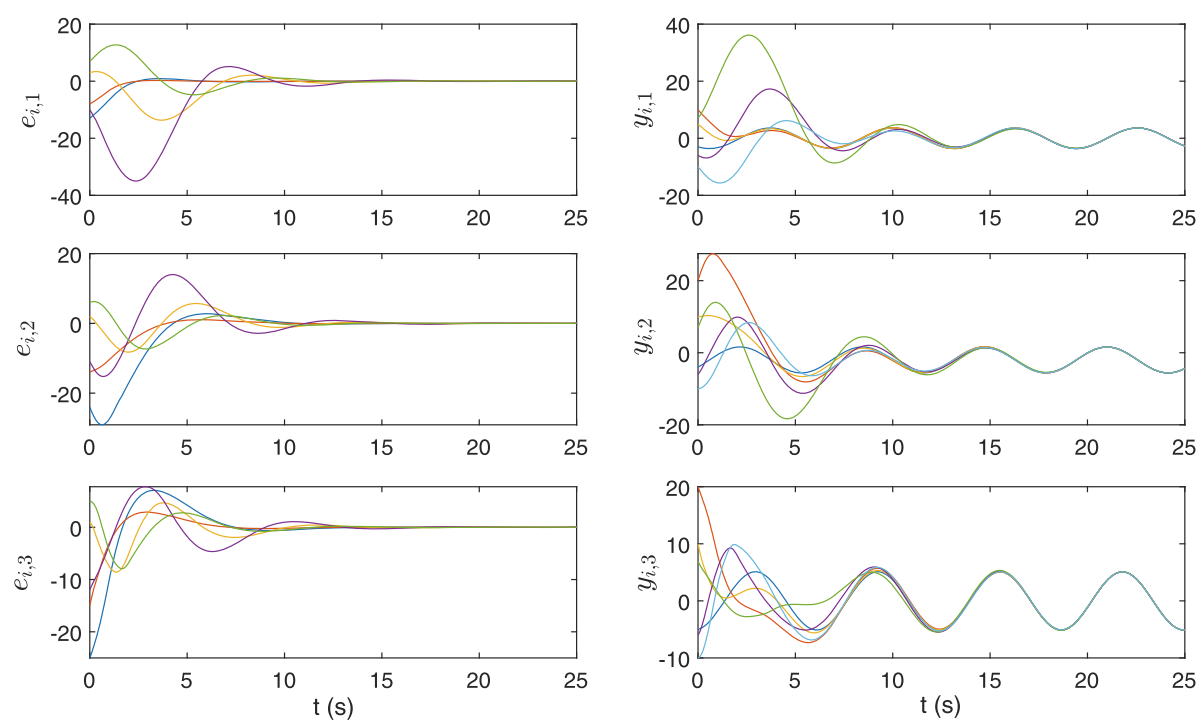

(A)

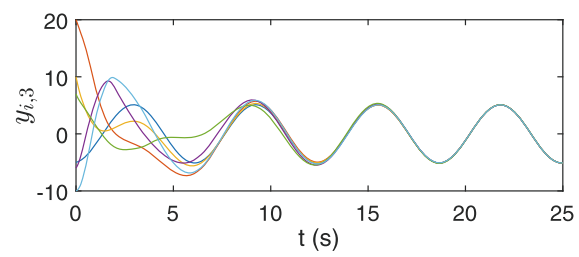

(B)

Fig. 3 Simulation results under the state feedback consensus protocols (9) with $\epsilon=0.1$. (A), Output consensus errors $e_{i}, i=1,2, \ldots, 5 ;$ (B), The outputs of the leader $y_{0}$ and the five followers $y_{i}, i=1,2, \ldots, 5$ 

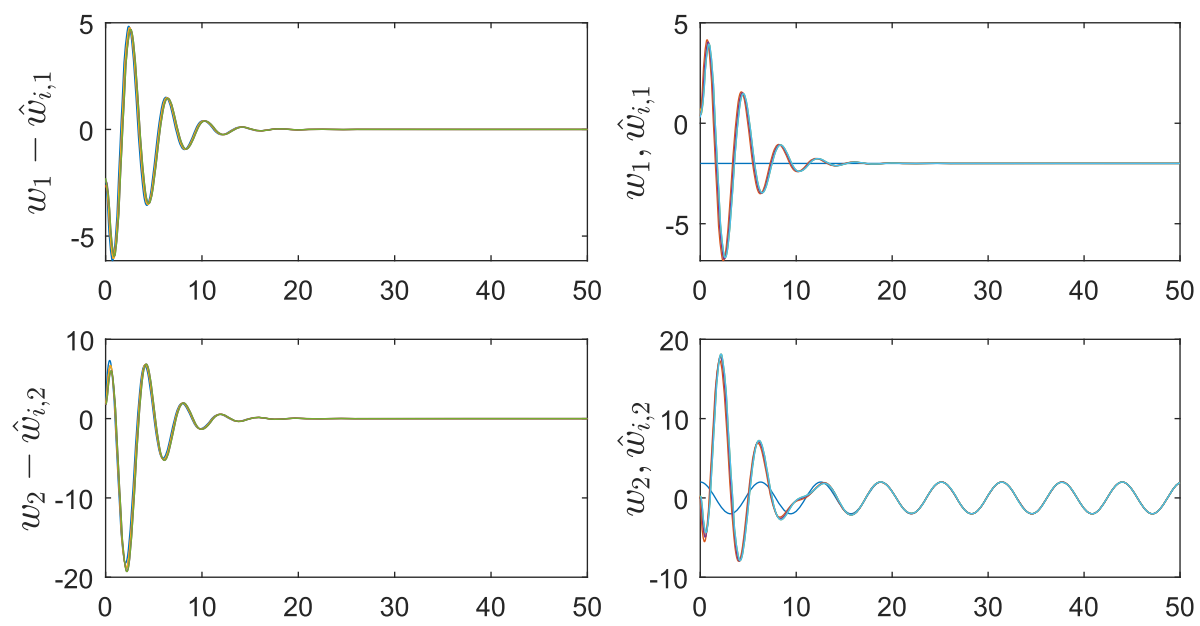

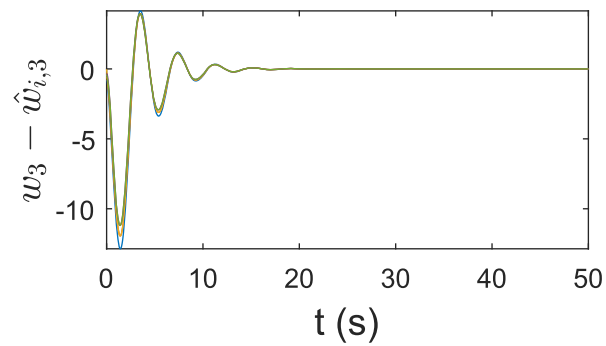

(A)

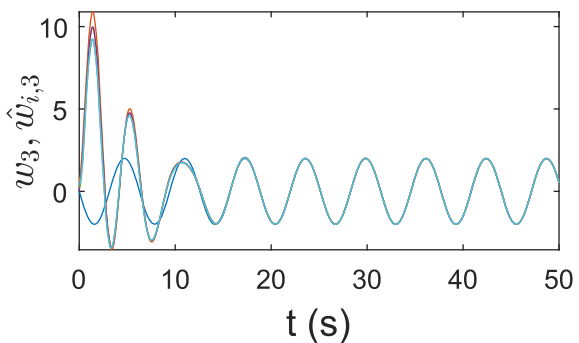

(B)

Fig. 4 (A), Elements of estimation errors $w-\hat{w}_{i}, i=1,2, \ldots, 5$; (B), Elements of the state of the leader $w$ and its estimations $\hat{w}_{i}, i=1,2, \ldots, 5$
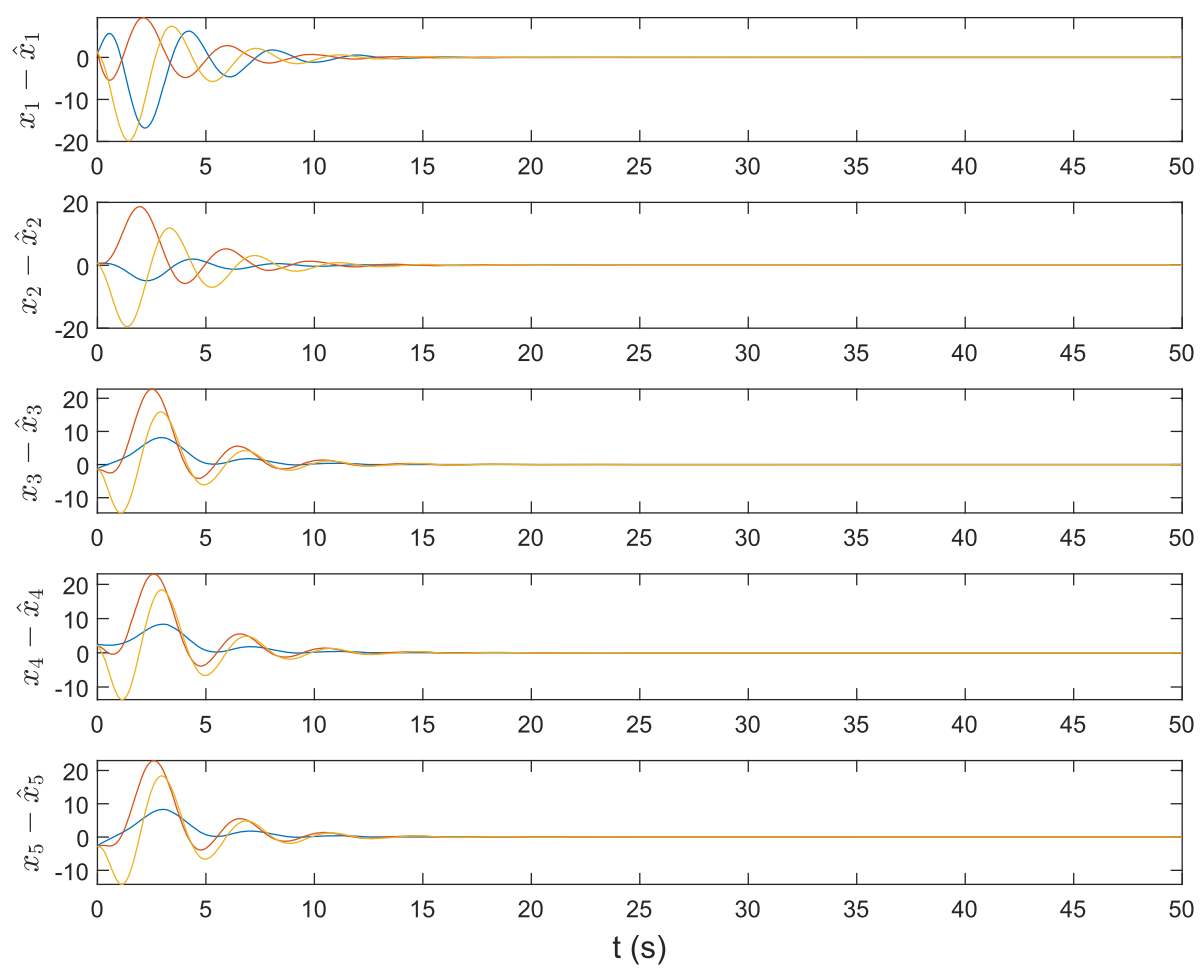

Fig. 5 Elements of estimation errors $x_{i}-\hat{x}_{i}, i=1,2, \ldots, 5$ 


$$
\begin{aligned}
\mathcal{L} & =\left[\begin{array}{cccccc}
0 & 0 & 0 & 0 & 0 & 0 \\
-1 & 2 & 0 & 0 & -1 & 0 \\
0 & -1 & 1 & 0 & 0 & 0 \\
0 & -1 & 0 & 1 & 0 & 0 \\
0 & 0 & 0 & -1 & 1 & 0 \\
0 & 0 & -1 & 0 & 0 & 1
\end{array}\right], \\
\mathcal{L}_{f f} & =\left[\begin{array}{ccccc}
2 & 0 & 0 & -1 & 0 \\
-1 & 1 & 0 & 0 & 0 \\
-1 & 0 & 1 & 0 & 0 \\
0 & 0 & -1 & 1 & 0 \\
0 & -1 & 0 & 0 & 1
\end{array}\right], \quad \mathcal{L}_{5}=\left[\begin{array}{cccc}
1 & 0 & 0 & 0 \\
0 & 1 & 0 & 0 \\
0 & -1 & 1 & 0 \\
-1 & 0 & 0 & 1
\end{array}\right] .
\end{aligned}
$$

Notice that all eigenvalues of $\mathcal{L}_{f f}$ and $\mathcal{L}_{5}$ have positive real parts.

The matrices of the leader are

$$
S=\left[\begin{array}{ccc}
0 & 0 & 0 \\
0 & 0 & 1 \\
0 & -1 & 0
\end{array}\right], \quad Q=\left[\begin{array}{ccc}
0 & -1 & 0 \\
-1 & 0 & -1 \\
0 & -1 & -1
\end{array}\right]
$$

For the followers 1 and 2, $i=1,2$, the matrices are

$$
\begin{aligned}
A_{i} & =\left[\begin{array}{ccc}
-1 & 0 & 0 \\
0 & 0 & 1 \\
0 & 0 & 0
\end{array}\right], B_{i}=\left[\begin{array}{ll}
1 & 0 \\
1 & 0 \\
0 & 2
\end{array}\right], W_{i}=\left[\begin{array}{ccc}
-1 & 0 & 1 \\
-1 & -1 & -1 \\
2 & -1 & 2
\end{array}\right], \\
C_{i} & =\left[\begin{array}{lll}
1 & 0 & 0 \\
0 & 1 & 1 \\
1 & 0 & 1
\end{array}\right] .
\end{aligned}
$$
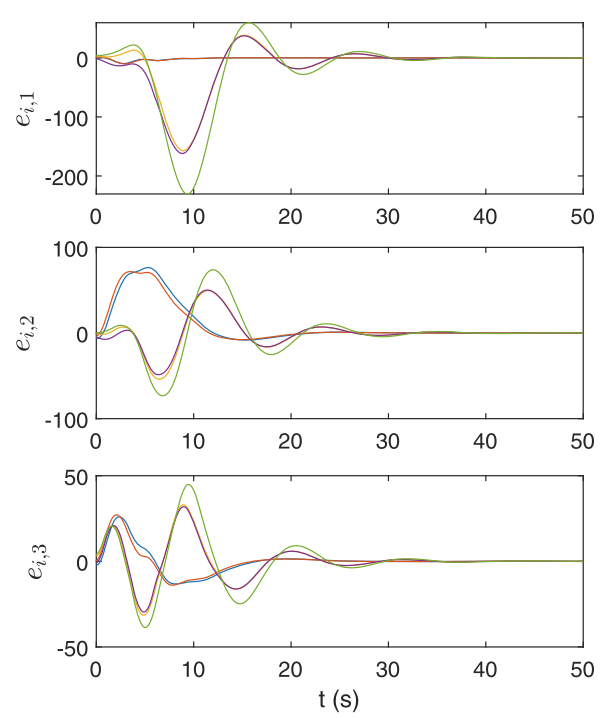

(A)
For the followers 3,4 and $5, i=3,4,5$, the system matrices are

$$
\begin{aligned}
A_{i} & =\left[\begin{array}{lll}
0 & 1 & 0 \\
0 & 0 & 1 \\
0 & 0 & 0
\end{array}\right], B_{i}=\left[\begin{array}{ll}
0 & 0 \\
0 & 1 \\
2 & 0
\end{array}\right], W_{i}=\left[\begin{array}{ccc}
-1 & 0 & 0 \\
-1 & -2 & -2 \\
0 & -3 & 3
\end{array}\right], \\
C_{i} & =\left[\begin{array}{lll}
1 & 0 & 0 \\
0 & 1 & 0 \\
0 & 0 & 1
\end{array}\right] .
\end{aligned}
$$

For $i=1,2, \ldots, 5$, all eigenvalues of $A_{i}$ have nonpositive real parts, and each pair of $\left(A_{i}, B_{i}\right)$ is stabilizable. We assume in this example that each follower has the same actuator "time constants", that is, for $i=1,2, \ldots, 5$, $\bar{T}_{i}=20 I_{3}$.

The solution of the regulator Eq. (3) gives

$$
\begin{array}{lll}
\Pi_{i}=\left[\begin{array}{lll}
0 & 1 & 0 \\
1 & 0 & 0 \\
0 & 0 & 1
\end{array}\right], & \Gamma_{i}=\left[\begin{array}{ccc}
1 & 1 & 0 \\
-1 & 0 & -1
\end{array}\right], \quad i=1,2, \\
\Pi_{i}=\left[\begin{array}{lll}
0 & 1 & 0 \\
1 & 0 & 1 \\
0 & 1 & 1
\end{array}\right], & \Gamma_{i}=\left[\begin{array}{ccc}
0 & 1 & -1 \\
1 & 0 & 1
\end{array}\right], \quad i=3,4,5 .
\end{array}
$$

The initial state of the leader is chosen as $w(0)=[-2-$ $3-2]^{\mathrm{T}}$. It follows that $\left\|\Gamma_{i} w(t)\right\| \leq 7.13$ and $\left\|\Gamma_{i} S w(t)\right\| \leq$ $5.84, i=1,2, \ldots, 5, \forall t \geq 0$. Let $p=10, r=10$. Thus, Assumption 5 is satisfied with $\delta_{p}=10, \delta_{r}=10$ and $T=0$.

\subsection{Semi-global output consensus via state feedback}

In this section, we present simulation results to verify state feedback consensus protocol (9) that solves Problem 1, under Assumptions 1-3 and 5.
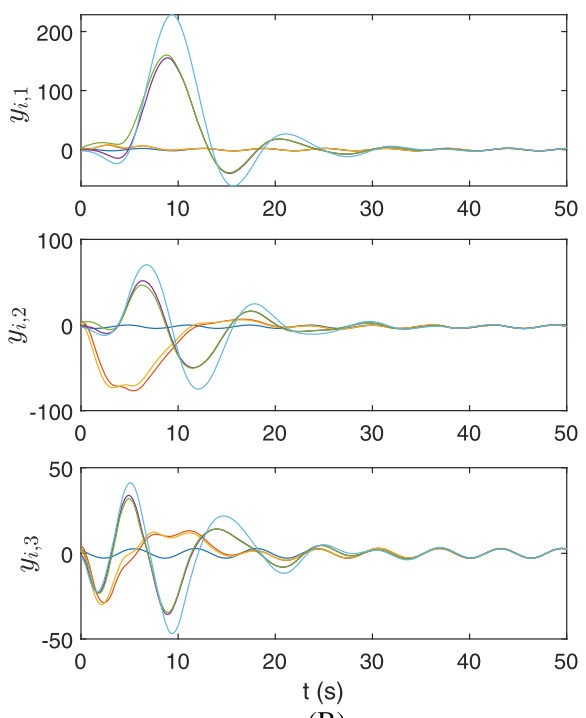

(B)

Fig. 6 Simulation results under the output feedback consensus protocols (21) with $\epsilon=0.01$. (A), Output consensus errors $e_{i}, i=1,2, \ldots, 5$; (B), The outputs of the leader $y_{0}$ and the five followers $y_{i}, i=1,2, \ldots, 5$ 
The initial states of the distributed observers (5) are set as random scalars between $(0,1)$ for $i=1,2, \ldots, 5$, and the positive scalar $\mu_{1}$ is chosen as $\mu_{1}=3$. It is easy to verify that $\left(I_{5} \otimes S-\mu_{1} \mathcal{L}_{f f} \otimes I_{3}\right)$ is Hurwitz. As shown in Fig. 2, the states $\eta_{i}(t)$ asymptotically converge to the state of the leader $w(t)$.

The initial system states and actuator positions of the followers are chosen as

$$
\begin{aligned}
& {\left[x_{1}(0) x_{2}(0) x_{3}(0) x_{4}(0) x_{5}(0)\right]=\left[\begin{array}{lllll}
10 & 5 & -6 & 7 & -10 \\
10 & 5 & -6 & 7 & -10 \\
10 & 5 & -6 & 7 & -10
\end{array}\right] \text {, }} \\
& {\left[\begin{array}{lllll}
v_{1}(0) & v_{2}(0) & v_{3}(0) & v_{4}(0) & v_{5}(0)
\end{array}\right]=\left[\begin{array}{lllll}
2 & -2 & 1 & -6 & -1 \\
2 & -2 & 1 & -6 & -1
\end{array}\right] \text {. }}
\end{aligned}
$$

The low gain parameter $\epsilon$ is chosen as $\epsilon=0.1$. The simulation result is shown in Fig. 3. It can be seen that the outputs of the five followers converge to the output of the leader asymptotically, and the output consensus errors asymptotically converge to 0 . Thus, the semi-global leader-following output consensus problem defined in Problem 1 is solved by the state feedback consensus control protocol (9) with $\epsilon=0.1$.

\subsection{Semi-global output consensus via output feedback} In this section, simulation results are given to verify output feedback consensus protocol (21) in solving Problem 2, under Assumptions 1-5.

For the informed follower 1 , i.e., $i=1$, the gain matrices in distributed observers (6) and (8) are set as $L_{A, 1}=$ $\operatorname{diag}\{-5,-6,-5\}$ and $L_{S, 1}=\left[\begin{array}{lll}1 & 1 & 0 \\ 0 & 1 & 0 \\ 0 & 0 & 2\end{array}\right]$. We can verify that $S+L_{S, 1} Q$ is Hurwitz. For the uninformed follower 2, i.e., $i=2$, the distributed observers (7) and (8) are constructed with $\mu_{2}=4, L_{A, 2}=L_{A, 1}$. For the rest uninformed followers i.e., $i=3,4,5$, we set $\mu_{2}=4, L_{A, i}=$ $\operatorname{diag}\{-2,-2,-2\}$. It follows that matrices $\left(I_{4} \otimes S-\mu_{2} \mathcal{L}_{5} \otimes\right.$ $\left.I_{3}\right)$ is all Hurwitz. For $i=1,2, \ldots, 5, A_{i}+L_{i} C_{i}$ are Hurwitz.

For $i=1,2, \ldots, 5$, the initial states of the distributed observers (6)-(8) are chosen as random constants between $(0,1)$. The initial system states and actuator positions of the followers are chosen as

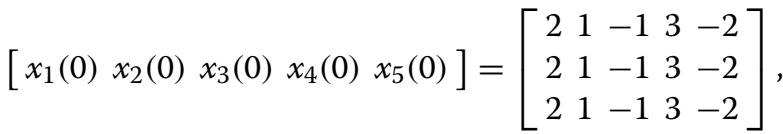

$$
\begin{aligned}
& {\left[\begin{array}{lllll}
v_{1}(0) & v_{2}(0) & v_{3}(0) & v_{4}(0) & v_{5}(0)
\end{array}\right]=\left[\begin{array}{lllll}
2 & -2 & 1 & 0 & -1 \\
2 & -2 & 1 & 0 & -1
\end{array}\right] \text {. }}
\end{aligned}
$$

The trajectories of $w_{i}, \hat{w}_{i}, x_{i}, \hat{x}_{i}$, and estimation errors $\hat{w}_{i}-w_{i}, \hat{x}_{i}-x_{i}, i=1,2, \ldots, 5$, are shown in Figs. 4 and 5 . It is clear that $\hat{w}_{i}$ converge to $w_{i}$ and $\hat{x}_{i}$ converge to $x_{i}$ asymptotically.

We consider low gain parameter $\epsilon=0.01$. It is easy to get solutions of the parametric ARE (4) with $\gamma=1$.
The simulation result is shown in Fig. 6, from which we can conclude that the outputs of the followers $y_{i}, i=$ $1,2, \ldots, 5$, are regulated to the output of the leader $y_{0}$ asymptotically and the output consensus errors $e_{i}$ converge to zero, which implies that the consensus protocol (21) achieves the semi-global leader-following output consensus problem with the followers subject to actuator position and rate saturation.

\section{Conclusion}

In this paper, we have investigated the semi-global output consensus problem for multiple heterogeneous linear systems subject to actuator position and rate saturation. Both a state feedback-based consensus protocol and an output feedback-based consensus protocol for each follower are constructed, using the information of the follower and its neighbors. It is proved that given any a priori given bounded conditions, the problem is solved by the consensus protocols if the low gain parameter is tuned small enough and the communication graph contains a spanning tree.

\section{Authors' contributions}

All authors contributed to the study conception and design. Mathematical analysis was performed by Panpan Zhou. The draft of the manuscript was written by Panpan Zhou, and the author Ben M. Chen commented on the previous versions of the manuscript and helped to revise the manuscript. All authors read and approved the final manuscript.

\section{Funding}

This paper was supported by the Research Grants Council of Hong Kong SAR (Grant No: 14209020).

Availability of data and materials

Not applicable.

\section{Code availability}

Not applicable.

\section{Declarations}

\section{Competing interests}

The authors declare no conflict of interest.

Received: 14 May 2021 Accepted: 19 September 2021

Published online: 14 October 2021

\section{References}

1. M. S. Radenković, M. Krstić, Distributed adaptive consensus and synchronization in complex networks of dynamical systems. Automatica. 91, 233-243 (2018)

2. Y. Wang, Y. Song, M. Krstić, C. Wen, Fault-tolerant finite time consensus for multiple uncertain nonlinear mechanical systems under single-way directed communication interactions and actuation failures. Automatica. 63, 374-383 (2016)

3. H. Meng, Z. Chen, R. Middleton, Consensus of multiagents in switching networks using input-to-state stability of switched systems. IEEE Trans. Autom. Control. 63(11), 3964-3971 (2018)

4. D. Li, S. S. Ge, T. Lee, Fixed-time-synchronized consensus control of multi-agent systems. IEEE Trans Control. Netw. Syst. 8(1), 89-98 (2020)

5. S. Dong, W. Ren, Z. Wu, Observer-based distributed mean-square consensus design for leader-following multiagent markov jump systems. IEEE Trans. Cybern. 51(6), 3054-3061 (2021) 
6. M. Lu, L. Liu, Leader-following attitude consensus of multiple rigid spacecraft systems under switching networks. IEEE Trans. Autom. Control. 65(2), 839-845 (2019)

7. Z. Li, W. Ren, X. Liu, L. Xie, Distributed consensus of linear multi-agent systems with adaptive dynamic protocols. Automatica. 49(7), 1986-1995 (2013)

8. Z. Li, G. Wen, Z. Duan, W. Ren, Designing fully distributed consensus protocols for linear multi-agent systems with directed graphs. IEEE Trans. Autom. Control. 60(4), 1152-1157 (2014)

9. Q. Ma, G. Miao, Output consensus for heterogeneous multi-agent systems with linear dynamics. Appl. Math. Comput. 271, 548-555 (2015)

10. T. Han, Z. Guan, B. Xiao, J. Wu, X. Chen, Distributed output consensus of heterogeneous multi-agent systems via an output regulation approach. Neurocomputing. 360, 131-137 (2019)

11. Y. Liu, H. Min, S. Wang, Z. Liu, S. Liao, Distributed consensus of a class of networked heterogeneous multi-agent systems. J. Frankl. Inst. 351(3), 1700-1716 (2014)

12. T. Liu, J. Huang, A distributed observer for a class of nonlinear systems and its application to a leader-following consensus problem. IEEE Trans. Autom. Control. 64(3), 1221-1227 (2018)

13. Y. Yu, Z. Li, Z. Yang, Z. Ding, Distributed back-stepping consensus protocol for attitude synchronization and tracking on undirected graphs. Unmanned Syst. 7(1), 25-32 (2019)

14. T. Yang, Z. Meng, D. V. Dimarogonas, K. H. Johansson, Global consensus for discrete-time multi-agent systems with input saturation constraints. Automatica. 50(2), 499-506 (2014)

15. L. Shi, Z. Lin, in Proceedings of the 37th Chinese Control Conference. Semi-global output containment control of linear multi-agent systems with actuator saturation, (2018), pp. 7160-7165

16. J. Qin, W. Fu, W. Zheng, H. Gao, On the bipartite consensus for generic linear multiagent systems with input saturation. IEEE Trans. Cybern. 47(8), 1948-1958 (2016)

17. L. Shi, Y. Li, Z. Lin, Semi-global leader-following output consensus of heterogeneous multi-agent systems with input saturation. Int. J. Robust Nonlinear Control. 28(16), 4916-4930 (2018)

18. L. Shi, Y. Li, Z. Lin, in Proceedings of the 2018 IEEE International Conference on Real-time Computing and Robotics. Semi-global leader-following output consensus of discrete-time linear multi-agent systems with input saturation, (2018), pp. 354-359

19. L. Shi, Y. Li, Z. Lin, in Proceedings of the 15th IEEE International Conference on Control and Automation. Semi-global output containment control for a group of heterogeneous discrete-time linear systems with input saturation, (2019), pp. 1138-1143

20. Z. Lin, Low Gain Feedback. (Springer-Verlag, London, 1999)

21. Y. Zheng, Y. Zhu, L. Wang, Consensus of heterogeneous multi-agent systems. IET Control. Theory Appl. 5(16), 1881-1888 (2011)

22. Z. Meng, Z. Zhao, Z. Lin, On global leader-following consensus of identical linear dynamic systems subject to actuator saturation. Syst. Control Lett. 62(2), 132-142 (2013)

23. Y. Xie, Z. Lin, in Proceedings of the 2019 Chinese Control Conference. Global consensus of multi-agent systems with intermittent directed communication in the presence of actuator saturation, (2019), pp. 5958-5963

24. M. Dornhein, Report pinpoints factors leading to YF-22 crash. Aviat. Week Space Technol. 9, 53-54 (1992)

25. Z. Lin, Semi-global stabilization of linear systems with position and rate-limited actuators. Syst. Control Lett. 30(1), 1-11 (1997)

26. Y. Lim, H. S. Ahn, Decentralized control of nonlinear interconnected systems under both amplitude and rate saturations. Automatica. 49(8), 2551-2555 (2013)

27. Z. Zhao, Z. Lin, Semi-global leader-following consensus of multiple linear systems with position and rate limited actuators. Int. J. Robust Nonlinear Control. 25(13), 2083-2100 (2015)

28. Z. Zhao, H. Shi, Semi-global containment control for linear systems in the presence of actuator position and rate saturation. Int. J. Robust Nonlinear Control. 29(1), 1-18 (2019)

29. Z. Lin, M. Pachter, S. Banda, Y. Shamash, in Guidance, Navigation, and Control Conference. Feedback design for robust tracking of linear systems with rate limited actuators, (1997), p. 3609

30. Y. Hong, X. Wang, Z. Jiang, Distributed output regulation of leader-follower multi-agent systems. Int. J. Robust Nonlinear Control. 23(1), 48-66 (2013)
31. Z. Li, W. Ren, X. Liu, M. Fu, Distributed containment control of multi-agent systems with general linear dynamics in the presence of multiple leaders. Int. J. Robust Nonlinear Control. 23(5), 534-547 (2013)

32. P. Zhou, B. M. Chen, in Proceedings of the 2021 Chinese Control Conference. Semi-global leader-following output consensus of heterogeneous systems with position and rate-limited actuators via state feedback, (2021), pp. 5295-5302

\section{Publisher's Note}

Springer Nature remains neutral with regard to jurisdictional claims in published maps and institutional affiliations. 